\title{
Recuperative Parametric Pumping in Adsorptive Membranes
}

\author{
Akhil Agrawal and Mark A. Burns \\ Dept. of Chemical Engineering, The University of Michigan, Ann Arbor, MI 48109
}

\begin{abstract}
Recuperative parametric pumping in adsorptive membranes, a cyclical adsorptive separation process, can lead to two distinct mechanisms of separation: rejection and preferential transport. During rejection, the adsorptive membrane rejects an adsorbing solute while permitting the transport of nonadsorbing solutes. During preferential transport the reverse occurs; the adsorptive membrane selectively transports an adsorbing solute while preventing the transport of nonadsorbing solutes. Switching from rejection to preferential transport can be accomplished by merely decreasing the ratio of the stroke volume (cyclical volume of solution pumped into and out of the membrane) to the membrane void volume. Preferential transport, earlier called an "inverse separation," results from the adsorbing solute crossing over from an adsorbing to a desorbing region in the interior of the membrane and is governed by the shape of the equilibrium isotherms and by process variables. In experiments with lysozyme and a membrane chromatography cartridge, selectivities obtained by preferential transport were on the order of 5 and agreed with theoretical predictions. Theoretical predictions suggest that to further improve separation by preferential transport, experiments need to be conducted on low dispersion membranes $\left(\mathrm{Pe}>10^{4}\right)$ and that both rejection and preferential transport can lead to continuous concentrated streams of the adsorbing solutes.
\end{abstract}

\section{Introduction}

Cyclic adsorptive separation processes can have many advantages over noncyclic adsorptive processes including higher selectivity, higher productivity, and continuous operation. For example, the cyclic adsorptive process pressure swing adsorption (PSA) is preferred over noncyclic adsorptive processes in many commercial gas-phase separations (Yang, 1987). PSA is particularly suitable for gas-phase separations because gas adsorption is a strong function of pressure. In addition, since the volume of gases significantly decreases with pressure, the amount of low-pressure desorbing gas required is small, leading to high productivity (Wankat, 1986). PSA does not offer similar advantages for liquid-phase separations because liquid-phase volumes are relatively constant over pressure.

For liquid-phase separations, parametric pumping is a promising cyclic adsorptive separation process. Parametric pumping has been studied by numerous investigators and several reviews on parametric pumping are available in the literature (Rice, 1976; Wankat, 1981; Sweed, 1984; Grevillot, 1986; Huang and Hollein, 1988). During parametric pumping, a packed bed is exposed to alternate adsorbing and non- adsorbing conditions synchronized to changes in flow direction. Under these conditions, separation occurs because adsorbing solutes migrate to one end of the packed bed while nonadsorbing solutes migrate to the other. This separation is essentially a result of the difference in the propagation velocities (velocities of solute fronts) of the adsorbing and nonadsorbing solutes under adsorbing conditions (Pigford, 1969).

The separation quality obtained depends on whether parametric pumping is conducted in the direct mode or in the recuperative mode. In the direct mode, the intensive variable (the variable that changes the adsorption isotherm) is controlled by externally imposing a change to the packed bed. For instance, in direct thermal parametric pumping, coils are wrapped around the bed to control the temperature in the bed. Thus, the change of the intensive variable (temperature) is independent of the flow of solutions. From the first studies, the direct mode has yielded exceptional results with separation factors (ratio of solute concentrations) of up to $10^{5}$ for a batch system (Wilhelm and Sweed, 1968) and $10^{3}$ for a continuous system (Chen et al., 1972). Since then, direct thermal 
parametric pumping has been extended to ionic mixtures (Butts et al., 1973), nonlinearly sorbing solutes (Camero and Sweed, 1976), multicomponent mixtures (Zhi and Wankat, 1983), and various applications other than adsorptive separations including liquid-liquid extraction (Wankat, 1973) and size exclusion chromatography (Koo and Wankat, 1988). The shortcomings of the direct mode are that direct thermal parametric pumping requires very high energy and that other desorbents, such as $\mathrm{pH}$ and ionic strength, are difficult to use (Wankat, 1981; Huang and Hollein, 1988).

An attractive alternative to the direct mode is the recuperative mode. In the recuperative mode, the intensive variable is controlled by imposing a change in the solution before it enters the column. This change may be accomplished in recuperative thermal parametric pumping by providing heat exchangers on each side of the bed. Thus, in contrast to the direct mode, change of the intensive variable in the recuperative mode is linked to the flow of the solutions. The recuperative mode of parametric pumping can be set up easily when $\mathrm{pH}$ and ionic strength are used as desorbents and can be up to fifteen times less energy intensive than the direct mode (Wankat, 1978).

Several researchers found that recuperative parametric pumping can lead to the rejection of the adsorbing solute and transport of the nonadsorbing solutes. This type of separation occurs when the volume of solvent displaced during the desorption step is larger than the void volume of the column. Sweed and Rigaudeau (1975) showed that high separation factors can only be obtained for large displacement volumes and that the poor separation factors obtained previously using recuperative parametric pumping (Wilhelm et al., 1966; Rolke and Wilhelm, 1969) were because they were conducted at low displacement volumes. Several studies that followed produced high separation factors for large displacement volumes and further confirmed this point (Almeida et al., 1982; Ferreira et al., 1991). Other studies of recuperative parametric pumping used 4-, 6-, or 8-step cycles and are difficult to compare to the two-step process (Chen et al., 1980; Chen et al., 1981; Hollein et al., 1982; Huang et al., 1985).

In some of the articles on the simple 2-step recuperative parametric pumping, a phenomenon called an "inverse separation" was observed (Sweed and Rigaudeau, 1975; Chen et al., 1977). This type of separation, which we term preferential transport, occurs when the volume of solvent displaced during the desorption step is less than the void volume of the column. For certain isotherms and experimental conditions, the adsorbing solute can be preferentially transported through the column while the nonadsorbing solute is retained by the column. So far, no definitive explanation on the methods for obtaining preferential transport has been presented (Grevillot, 1986).

This study focuses on understanding the phenomenon of preferential transport and explaining the conditions necessary to obtain it. In addition, this study extends the theory of recuperative parametric pumping to adsorptive membranes. As far as we are aware, there have been no previous studies on recuperative parametric pumping in adsorptive membranes. (Studies in cyclic electrodialysis, which is an interesting variant of direct parametric pumping, were performed on ion-selective membranes (Thompson and Bass, 1974)). Adsorptive membranes may be more suitable than packed beds for recuperative parametric pumping because they offer lower mass-transfer resistances than packed beds (Brandt et al., 1988), a problem known to limit separation factors (Chen et al., 1981). Moreover, preferential transport in adsorptive membranes is particularly interesting because this phenomenon can be used for simultaneous size-based and adsorption-based separations. In such separations, solutes or particles larger than the membrane pore size are retained by the membrane with the nonadsorbing solutes while an adsorbing solute is transported across the membrane.

We will first demonstrate the phenomena of rejection and preferential transport theoretically and experimentally for a system consisting of the protein lysozyme, the desorbent $\mathrm{KCl}$, and a membrane chromatography cartridge. Next, we will describe methods for obtaining preferential transport and explore the effects of process variables on fluxes that are obtained during preferential transport. Finally, we will present strategies for operating continuous rejection and preferential transport to obtain continuous streams of an adsorbing solute.

\section{Model Development and Solution}

To theoretically study recuperative parametric pumping in adsorptive membranes, the unsteady-state mass balances of all solutes as well as the desorbent must be written. For this cyclic process, the unsteady-state movement must be modeled for both the adsorbing stroke and the desorbing stroke of each cycle until coherence, described by Helfferich (1986) as the state that such a system strives to attain, is attained. At coherence, the solute movement and concentration profiles do not change from one cycle to the next.

In the model, the membrane is considered isothermal and homogeneous with a uniform cross-sectional area $A$, porosity $\epsilon$, and a thickness $L$. The assumption of homogeneity is valid for thick membranes that are made from stacks of thinner membranes (Suen and Etzel, 1992). The system is assumed to be one-dimensional in the direction of flow with the direction of the adsorbing stroke being positive. The mass balances of a solute $i$ and of the desorbent $d$ (if the adsorption of the desorbent is negligible) for the adsorbing stroke (first half of a cycle) are given by Eqs. 1 and 2, respectively.

$$
\begin{gathered}
\frac{\partial C_{i}}{\partial t}+\frac{1}{\epsilon} \frac{\partial Q_{i}}{\partial t}=E_{i} \frac{\partial^{2} C_{i}}{\partial^{2} l}-u_{a} \frac{\partial C_{i}}{\partial l} \\
\frac{\partial C_{d}}{\partial t}=E_{d} \frac{\partial^{2} C_{d}}{\partial^{2} l}-u_{a} \frac{\partial C_{d}}{\partial l},
\end{gathered}
$$

where $E_{i}$ and $E_{d}$ are the axial dispersion coefficients for species $i$ and desorbent, respectively, and $u_{a}$ is the interstitial velocity during the adsorbing stroke. The mass balances for the desorbing stroke are also given by Eqs. 1 and 2, except that the velocity $u_{a}$ is replaced by velocity $u_{d}$ and the dispersion coefficients are evaluated at the velocity $u_{\mathrm{j}}$. In Eqs. 1 and 2, it has been assumed that the axial dispersion coefficient is not enhanced by the changes in flow direction. This assumption is valid when the characteristic time for diffusion across the pore radius $\left(r^{2} / D\right)$ is low compared to the 
cycle time $\left(T_{c}\right)$ (Leighton and McCready, 1988), a condition met in our system. Also, the flux of solute due to electric potential gradients has been neglected. This flux would be important for ion-selective membranes used in cyclic electrodialysis because such membranes have small pore diameters $(\approx 60 \AA$ ) (Guzman-Garcia et al., 1990), but this flux is negligible in our ion-exchange membrane because the pores are $\sim 1 \mu \mathrm{m}$.

Equations 1 and 2 are coupled by an adsorption isotherm that includes the effect of the desorbent. A suitable isotherm is the Langmuir isotherm because of its wide applicability. The Langmuir isotherm for single-component systems is given by

$$
Q_{i}=\frac{Q_{m, i} K_{m, i} C_{i}}{1+K_{m, i} C_{i}}
$$

where the adsorbent capacity, $Q_{m, i}$, and equilibrium constant, $K_{m, i}$, are functions of the desorbent concentration. These functions can be written (Antia and Horvath, 1989)

$$
\begin{aligned}
& K_{m, i}=K_{m, i}^{\max } f\left(C_{d}\right) \\
& Q_{m, i}=Q_{m, i}^{\max } g\left(C_{d}\right),
\end{aligned}
$$

where $C_{d}$ is the concentration of the desorbent, $K_{m, i}^{\max }$ and $Q_{m, i}^{\max }$ are the values of equilibrium constant and membrane capacity of solute $i$, respectively, at zero desorbent concentration, and $f$ and $g$ are experimentally determined functions. In Eq. 3, it is assumed that desorption is induced by effects other than competitive binding; for systems in which competitive binding is important, Eqs. 3, 4a and $4 \mathrm{~b}$ can be replaced by isotherms that account for competitive binding (Brooks and Cramer, 1992). Additionally, it is assumed that local equilibrium exists between the solid and liquid phases, a valid assumption for adsorptive membranes under a variety of conditions (Suen and Etzel, 1992; Briefs and Kula, 1992). In particular, the assumption of local equilibrium is consistent with the results of a study conducted with ion-exchange membranes and liquid velocity values similar to those used in this study (Weinbrenner and Etzel, 1994).

The initial condition for Eqs. 1 and 2 is given by

$$
C_{i}=0 \quad \text { and } \quad C_{d}=0 \quad \text { at } \quad t=0,0 \leq l \leq L .
$$

The boundary conditions change after each half-cycle since the direction of flow is reversed. For the adsorbing stroke they are given by (Danckwerts, 1952):

$$
\begin{gathered}
\text { for }(n-1) T_{c}<t \leq(n-1) T_{c}+\frac{T_{c}}{2}: \\
u_{a} C_{i, 0}=u_{a} C_{i}+E_{i}\left(\frac{\partial C_{i}}{\partial l}\right) \text { at } l=0 \\
\left(\frac{\partial C_{i}}{\partial l}\right)=0 \quad \text { at } \quad l=L .
\end{gathered}
$$

Similarly, for the desorbing stroke they are given by:

$$
\begin{aligned}
& \text { for }(n-1) T_{c}+\frac{T_{c}}{2}<t \leq n T_{c}: \\
& \qquad\left(\frac{\partial C_{i}}{\partial l}\right)=0 \text { at } l=0 \\
& 0=u_{d} C_{i}+E_{i}\left(\frac{\partial C_{i}}{\partial l}\right) \text { at } l=L,
\end{aligned}
$$

where $n$ is the cycle number, and $T_{c}$ is the cycle time. Note that the desorbent is introduced from an opposite side of the membrane from the solute $i$. Therefore, the boundary conditions for the desorbent are the appropriate variations of Eqs. $6,7,8$, and 9 .

The equations in the mathematical model (Eqs. 1-9) were dedimensionalized and then solved using finite difference methods. The dimensionless variables are

$$
\begin{gathered}
\tau_{\alpha}=\frac{u_{a} t}{L} \quad \tau_{d}=\frac{u_{d} t}{L} \quad z=\frac{l}{L} \quad x=\frac{C_{d}}{C_{d, 0}} \\
y_{i}=\frac{C_{i}}{C_{i, 0}} \quad y_{s, i}=\frac{Q_{i}}{Q_{m, i}^{\max }} \\
v_{i}=\frac{K_{m, i}}{K_{m, i}^{\max }} \quad w_{i}=\frac{Q_{m, i}}{Q_{m, i}^{\max }}
\end{gathered}
$$

where $\tau_{\alpha}$ and $\tau_{d}$ are dimensionless time during the absorbing and desorbing strokes, respectively, $z$ is the dimensionless axial position, $y_{i}$ and $x$ are dimensionless concentrations of the solute $i$ and desorbent, respectively, and $y_{s, i}$ is dimensionless concentration of the solute $i$ on the surface. After dedimensionalization, Eqs. 1-9 are given by

$$
\begin{gathered}
\frac{\partial y_{i}}{\partial \tau_{a}}+\delta_{i} \frac{\partial y_{s, i}}{\partial \tau_{a}}=\frac{1}{P e_{i}} \frac{\partial^{2} y_{i}}{\partial^{2} z}-\frac{\partial y_{i}}{\partial z} \\
\frac{\partial x}{\partial \tau_{a}}=\frac{1}{P e_{x}} \frac{\partial^{2} x}{\partial^{2} z}-\frac{\partial x}{\partial z} \\
y_{s, i}=\frac{\alpha_{i} v_{i} w_{i} y_{i}}{1+\alpha_{i} v_{i} y_{i}} \\
v_{i}=f(x) \\
w_{i}=g(x)
\end{gathered}
$$

for a cycle $n, 0<\tau_{a} \leq \frac{V_{s, a}}{V_{m}}$

$$
y_{i, 0}=y_{i}+\frac{1}{P e_{i}}\left(\frac{\partial y_{i}}{\partial z}\right) \text { at } z=0
$$

$$
\left(\frac{\partial y_{i}}{\partial z}\right)=0 \quad \text { at } \quad z=1
$$

for a cycle $n, \frac{V_{s, a}}{V_{m}}<\tau_{d} \leq \frac{V_{s, d}}{V_{m}}$

$$
\begin{gathered}
\left(\frac{\partial y_{i}}{\partial z}\right)=0 \quad \text { at } \quad z=0 \\
y_{i, 0}=y_{i}+\frac{1}{P e_{i}}\left(\frac{\partial y_{i}}{\partial z}\right) \quad \text { at } \quad z=1
\end{gathered}
$$


Table 1. Dimensionless Parameters

$\begin{array}{ll}P e_{i}=\frac{u_{a} L}{E_{i}} & \text { Peclet number for species } i \\ \alpha_{i}=K_{m, i}^{\max } C_{i, 0} & \text { Isotherm linearity parameter for species } i \\ \delta_{i}=\frac{Q_{m, i}^{\max }}{\epsilon C_{i, 0}} & \text { Dimensionless membrane capacity for species } i \\ \frac{V_{s, a}}{V_{m}}=\frac{u_{a} T_{i}}{2 L} & \text { Dimensionless adsorbing stroke volume } \\ \frac{V_{s, u}}{V_{s, d}}=\frac{u_{a}}{u_{d}} & \begin{array}{c}\text { Ratio of adsorbing stroke volume to desorbing } \\ \text { stroke volume }\end{array}\end{array}$

Note that $\tau_{a}$ in Eqs. 10 and 11 is replaced by $\tau_{d}$ for the desorbing half-cycle and that the boundary conditions for the desorbent are the appropriate variants of Eqs. 15-18. The dedimensionalization leads to the dimensionless parameters given in Table 1.

Equations 10-18 constitute a set of nonlinear coupled partial differential equations with associated initial and boundary conditions. These equations were solved numerically using finite difference techniques, the choice of which depended on the value of the Peclet number $(\mathrm{Pe})$. For $\mathrm{Pe}$ equal to infinity (plug flow) an explicit backward differencing method was used. For $P e>10^{3}$ the explicit Dufort Frankel method was used; this method is more accurate than the widely used classic explicit method (Lapidus and Pinder, 1982). For $P e<10^{3}$, an implicit state variable method (McCracken et al., 1970) was used; this method is free of oscillations for $0<P e \leq 10^{3}$ when both the convection and dispersion terms are important. Additionally, in this range of $P e$, the implicit state variable method is more stable than other methods, such as the Crank-Nicolson and the centered difference methods that are commonly used to solve such problems (Ramirez, 1989). Note that the solutions of the explicit Dufort Frankel method for $P e>10^{3}$ and the implicit state variable method for $P e<10^{3}$ matched at $P e=10^{3}$.

For each finite difference method a FORTRAN program was developed and the programs were run on IBM RS6000 and SUN Sparcstation computers. The numerical method parameters, which are the dimensionless spatial step increment and the dimensionless temporal step increment, were chosen based on the criterion that the numerical solution of Eq. 11 matched the analytical solution (Lapidus and Amundson, 1952) of Eq. 11.

\section{Materials and Methods Materials}

Lysozyme (L 6876), myoglobin (M 1882), and sodium azide (S 2002) were purchased from Sigma Chemical Company (St. Louis, MO). Potassium chloride (3040-01), dibasic anhydrous sodium phosphate (3828-01), and concentrated hydrochloric acid (9535-01) were purchased from J. T. Baker Inc. (Phillipsburg, NJ). Water was distilled in a Barnstead (Boston, MA) glass still (A 1040) and deionized in a Barnstead Nanopore II deionizer (D 3700). The buffer used in all the experiments was $0.02 \mathrm{M} \mathrm{Na}_{2} \mathrm{PO}_{4}$ at $\mathrm{pH} 7.5$. The membrane chromatography cartridge (CICM10H01) was purchased from Millipore Corporation (Bedford, MA). This cartridge is a stack of cation-exchange membranes with a bed volume of $1.4 \mathrm{~mL}$
$(0.5 \times 1.9 \mathrm{~cm}$ ID) and a porosity of 0.825 . The cartridge provides a $1.2-\mu \mathrm{m}$ macroporous network of cellulose modified with carboxymethyl groups with an ion-exchange capacity of about $0.68 \mathrm{meq}$ (manufacturer's data). All solutions were filtered using a $0.22-\mu \mathrm{m}$ Durapore filter (GVWP 04700 ) also purchased from Millipore Corporation.

\section{Determination of solute concentration}

The concentrations of lysozyme and myoglobin were determined by measuring absorbance at $280 \mathrm{~nm}$ and $410 \mathrm{~nm}$, while the concentrations of $\mathrm{KCl}$ were determined by measuring conductivity on a VWR Scientific Digital Conductivity Meter (Model 604). The spectrophotometer used for measuring protein concentration in batch quantities was a HP 8452A diode array spectrophotometer equipped with a cell of path length $10 \mathrm{~mm}$ and chamber volume $1 \mathrm{~mL}$. The monitors used for continuous measurements of protein concentration were Pharmacia UV-1 monitors equipped with flow cells of path length $10 \mathrm{~mm}$ and chamber volume $8.7 \mu \mathrm{L}$.

\section{Determination of dynamic adsorption isotherms}

Before the determination of adsorption isotherms, the total system void volume (consisting of the membrane, UV monitor, and intermediate tubing void volumes) was determined by measuring the breakthrough of lysozyme in $0.5 \mathrm{M}$ $\mathrm{KCl}$ solution. The determination of adsorption isotherms then proceeded as follows. First, the membrane cartridge was equilibrated with the buffer solution. Next, lysozyme in pure buffer was fed through the membrane cartridge and the emerging solvent was collected until the exit concentration reached $50 \%$ of the feed concentration. The dynamic capacity of the membrane was determined by subtracting the total system void volume from the volume of solvent collected and multiplying the result by the feed concentration. Dynamic capacities were determined for several protein concentrations to yield an adsorption isotherm. Such an adsorption isotherm was also determined for lysozyme in several $\mathrm{KCl}$ concentrations.

\section{Recuperative parametric pumping experiments}

The recuperative parametric pumping apparatus consisted of the membrane cartridge, two syringe pumps, two 3-way solenoid-actuated pinch valves (G-98301-22), two 2-way solenoid-actuated pinch values (G-98301-01), a time-delay relay (G-20602-30), two UV monitors connected to a computer, and four beakers (Figure 1). The syringe pumps (models 352 and 355) were purchased from Orion Research Inc. (Boston), while the solenoid valves and the time-delay relay were purchased from Cole-Parmer Instrument Co. (Chicago). The four solenoid valves were controlled by the time-delay relay that could be set to a particular cycle time $T_{c}$.

Before the experiment was begun, the membrane, the UV monitors, and all the tubing were equilibrated with the buffer solution. The experiment then proceeded as follows. During the first half cycle, protein flowed through the membrane and the right UV monitor into a beaker, while the desorbent flow was diverted into a beaker (effectively shutting off the desorbent pump). During the second half-cycle, desorbent flowed through the membrane and the left UV monitor and 


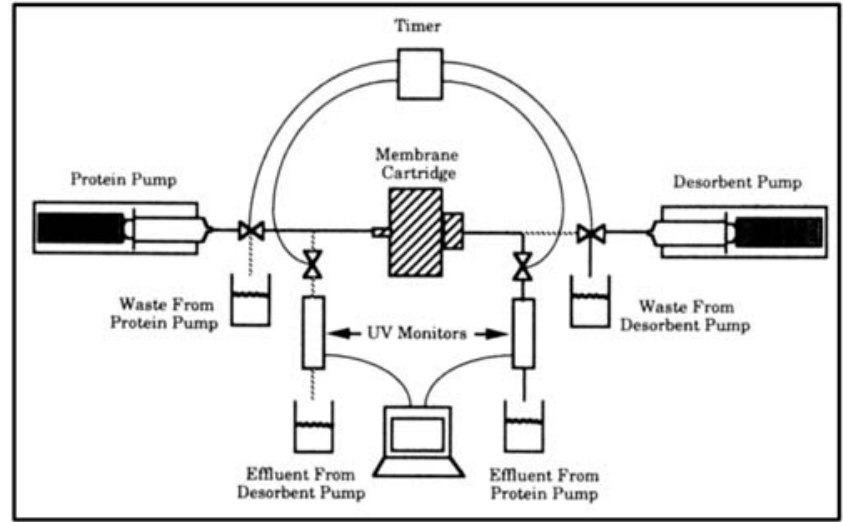

Figure 1. Recuperative parametric pumping apparatus.

During the first half cycle, protein flows through the membrane and the right UV monitor into a beaker, while the desorbent flow is diverted into a waste beaker (shown by dark lines). During the second half cycle, desorbent flows through the membrane and past the left UV monitor into a beaker, while the protein flow is diverted to a waste beaker.

into a beaker while the protein flow was diverted to a beaker (effectively shutting off the protein pump). These cycles were continued until coherence was attained. At coherence, the amount of protein fed in a cycle was equal to the amount of protein collected in the two effluent beakers in that cycle; such a state was attained after 10-60 cycles, depending on the experimental parameters. The protein concentrations in the effluent streams were measured by the UV monitors, and the amount of protein collected during each half cycle was determined by calculating the areas under the measured concentration profiles. This technique was verified by mixing each solution collected and then measuring the concentration on the diode array spectrophotometer.

The experiments just described were conducted under several different conditions. Stroke volumes were varied by changing the cycle times $\left(T_{c}\right)$ on the timer. Asymmetric strokes were obtained by keeping the flow rate of the protein pump constant $(0.6 \mathrm{~mL} / \mathrm{min})$ while changing the flow rate of the $\mathrm{KCl}$ pump. The protein solutions used in the experiments were lysozyme $(4.0 \mathrm{mg} / \mathrm{mL})$ in adsorbing conditions $(0.0 \mathrm{M}$ $\mathrm{KCl}$ in buffer), lysozyme $(4.0 \mathrm{mg} / \mathrm{mL})$ in nonadsorbing conditions $(0.35 \mathrm{M} \mathrm{KCl}$ in buffer), and a mixture of lysozyme $(4.0$ $\mathrm{mg} / \mathrm{mL})$ and myoglobin $(1.0 \mathrm{mg} / \mathrm{mL})$ in adsorbing conditions (0.0 $\mathrm{M} \mathrm{KCl}$ in buffer).

\section{Membrane cleaning and storage}

After each experiment, the membrane was rinsed with 0.5 $\mathrm{M} \mathrm{KCl}$ until there was no detectable protein in the eluent. Typically, this occurred after flowing about $30 \mathrm{~mL}$ of $0.5 \mathrm{M}$ $\mathrm{KCl}$. At the end of the day, the membrane cartridge was flushed with $25 \mathrm{~mL}$ distilled and deionized water and then with $10 \mathrm{~mL}$ of a $0.02 \%$ sodium azide solution in deionized water before storage at $4^{\circ} \mathrm{C}$.

\section{Error analysis}

Fractional flux measurements were made in each experiment for a minimum of five cycles after coherence was attained. Pump flow rate variations were quantified by several $(\sim 10)$ independent recuperative parametric pumping experiments. For these fractional flux and pump flow rate measurements, mean values and standard deviations were calculated.

\section{Results and Discussion}

In recuperative parametric pumping, two distinct modes of adsorbing-solute transport are possible: rejection and preferential transport. In this section, we first show the theoretical solute profiles for both rejection and preferential transport and then compare the predicted theoretical fluxes with experimental fluxes. Since the phenomenon of preferential transport is more complicated than rejection, methods for obtaining preferential transport are next outlined, and the influence of each process variable on preferential transport is given. Finally, the performance of continuous separations using rejection and preferential transport is evaluated.

\section{Rejection and preferential transport}

Rejection occurs when the displacement volume, also referred to as the stroke volume $\left(V_{s}\right)$, is greater than the membrane void volume $\left(V_{m}\right)$. Solute movement during rejection is described in Figure 2. In the first half of a cycle, an adsorbing solution containing an adsorbing solute $A$ and a nonadsorbing solute $B$ is fed through the membrane. During this time, $A$ adsorbs onto the surface of the membrane while $B$ passes through the membrane. In the second half of the cycle, $A$ desorbs and is transported to the left side of the membrane by a solute-free desorbing solution. In this way, the adsorbing solute is retained by the membrane while the nonadsorbing solute is transported through the membrane.

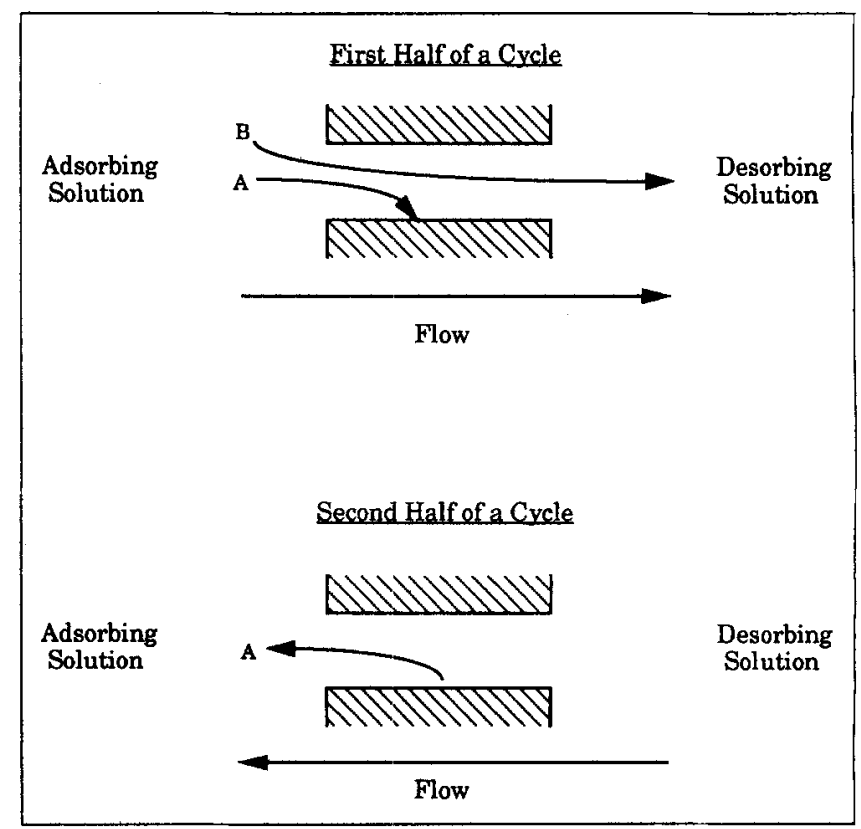

Figure 2. Mechanism of separation in rejection.

In the first half of a cycle, the adsorbing solute $A$ adsorbs while the nonadsorbing solute $B$ flows through. In the second half of a cycle, the adsorbing solute is "rejected" by the membrane because of desorbent breakthrough. 

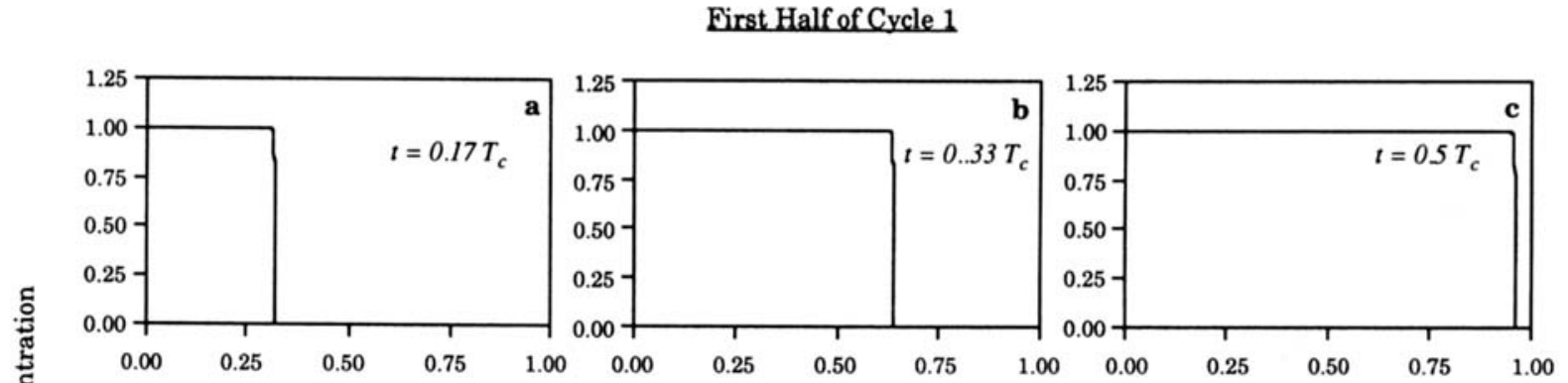

\section{Second Half of Cycle 1}
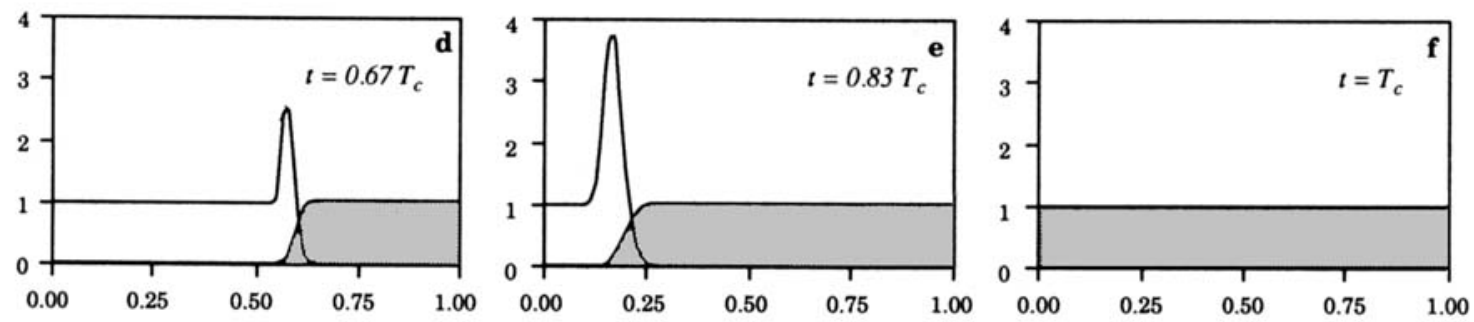

Dimensionless Distance

\section{Figure 3. Simulated concentration profiles of the adsorbing solute in rejection.}

(a)-(c) During the first half of cycle 1 (flow is to the right), the adsorbing solute wave (shown as a single curve) remains in the membrane. (d)-(f) During the second half of cycle 1 (flow is to the left), the desorbent wave (shown as shaded curve) breaks through the membrane leading to the "rejection" of the adsorbing solute. In the simulation, $V_{s} / V_{m}=1.2, Q_{m}=1.0 \mathrm{mg} / \mathrm{mL}$; all other parameters are given in Table

In order to verify the movement of solutes shown in Figure 2 , solute movement during rejection was simulated using the mathematical model. The concentration profiles, or waves, of the adsorbing solute and of the desorbent are shown in Figure 3 for the first cycle. In this particular simulation, $V_{s} / V_{m}$ is equal to 1.2 and the membrane is initially equilibrated with an adsorbing, solute-free solution. During the first half-cycle, the adsorbing solute wave remains within the membrane. At the end of this half-cycle, however, approximately a third of the nonadsorbing solute fed into the membrane is transported to the right. During the second half of the cycle, the desorbent wave breaks through the membrane, leading to solute desorption and subsequent transport of the adsorbing solute to the left of the membrane. Thus, with the stroke volume greater than the membrane void volume, the adsorbing solute is "rejected" by the membrane and remains on the left side.

Preferential transport, on the other hand, occurs when the stroke volume is less than the membrane void volume. Solute movement during preferential transport is described in Figure 4 . In the first half of the cycle, the adsorbing solute $A$ adsorbs to the surface of the membrane while the nonadsorbing solute $B$ remains in the membrane void. In the second half of the cycle, a solute-free desorbing solution is fed into the membrane and $B$ is transported back to the membrane's left, while $A$ desorbs but stays within the desorption front. This desorbed $A$ is transported across the membrane in the first half of the next cycle. In this way, the nonadsorbing solute is "rejected" by the membrane while the adsorbing solute is preferentially transported through the membrane.

The predicted adsorbing solute and desorbent waves dur-

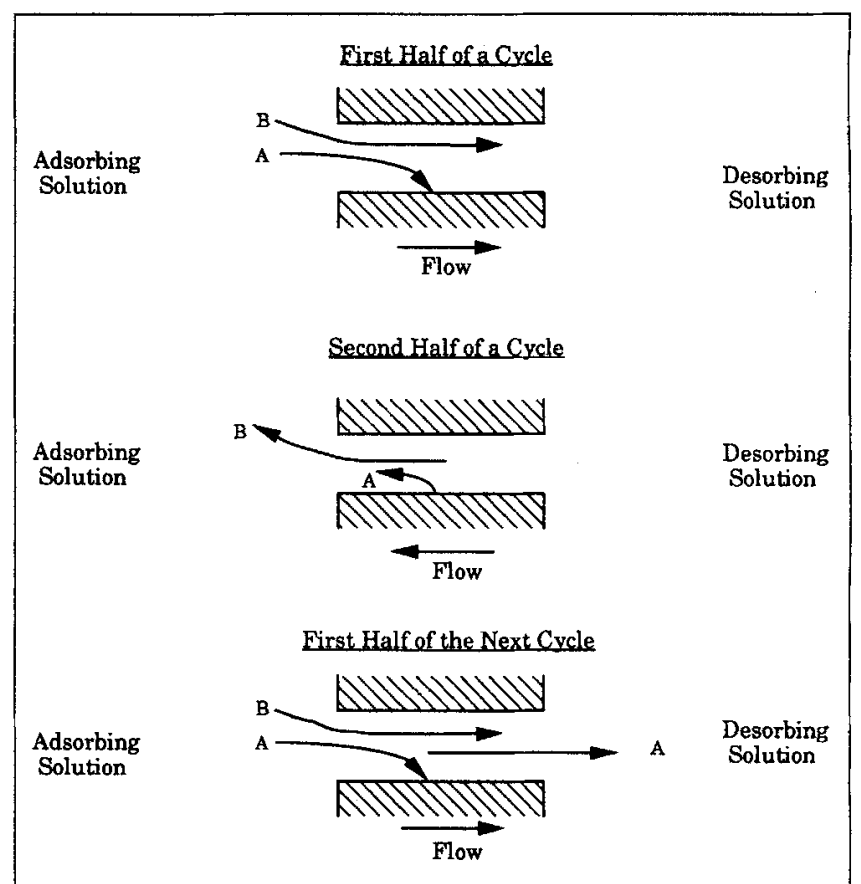

Figure 4. Mechanism of separation in preferential transport.

In the first half of the cycle, the adsorbing solute $A$ adsorbs to the membrane surface, while the nonadsorbing solute $B$ remains in the membrane void. In the second half of the cycle, $B$ is "rejected" by the membrane while $A$ desorbs but stays within the desorption front. In the first half of the next cycle, this desorbed $A$ is preferentially transported across the membrane. 
First Half of Cycle 1
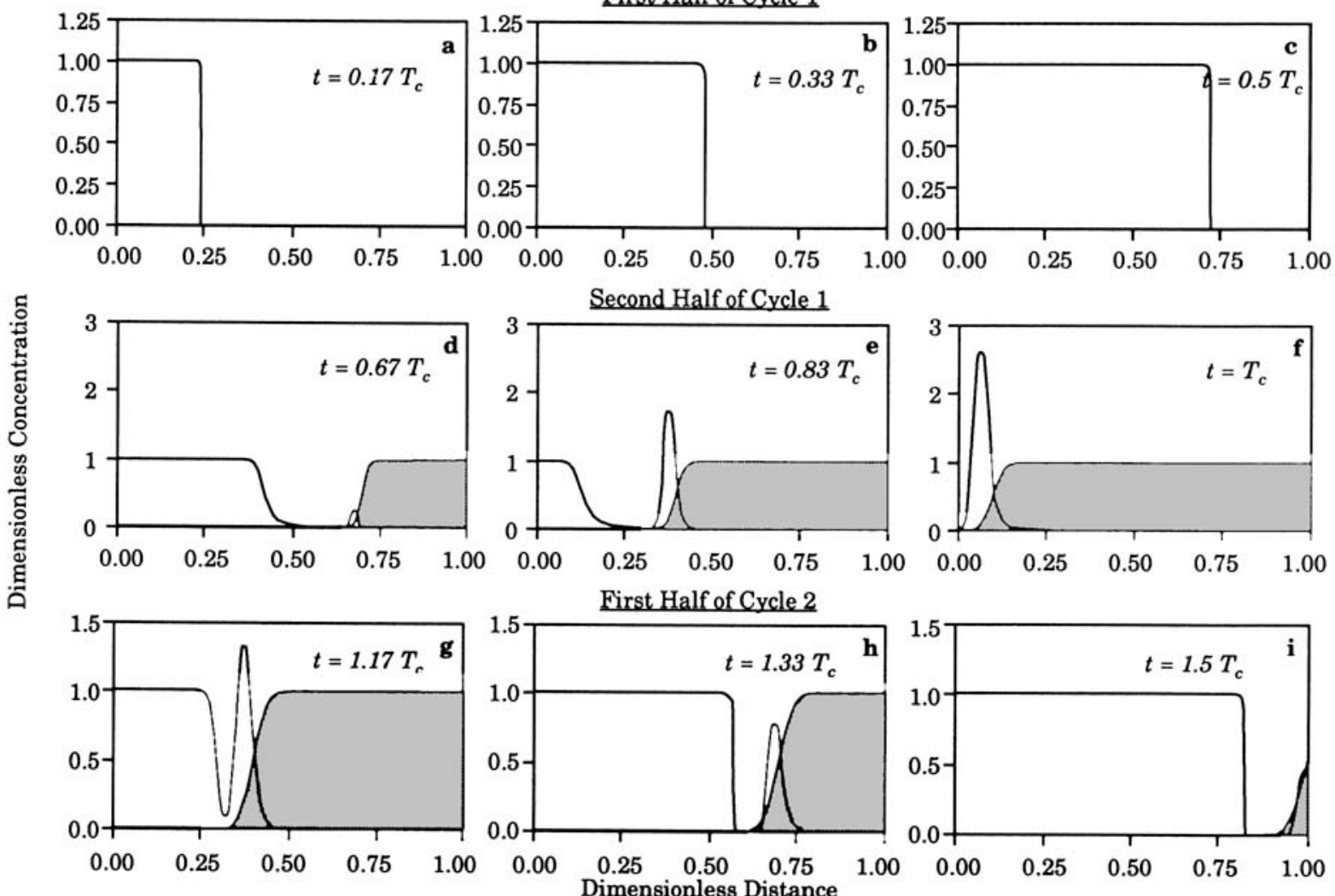

Figure 5. Simulated concentration profiles of the adsorbing solute in preferential transport.

(a)-(c) During the first half of cycle 1 (flow is to the right), the adsorbing solute wave (shown as a single curve) remains in the membrane. (d)-(f) During the second half of cycle 1 (flow is to the left), the desorbed solute forms a peak around the front of the desorbent wave (shown as shaded curve). ( $\mathrm{g}-\mathrm{i}$ ) During the first half of cycle 2, a fraction of the peak is transported to the right. In the simulation, $V_{s} / V_{m}=0.9, Q_{m}=1.0 \mathrm{mg} / \mathrm{mL}$; all other parameters are given in Table 2 .

ing preferential transport are shown in Figure 5 for the first one-and-a-half cycles. In this particular simulation $V_{s} / V_{m}$ is equal to 0.9 and the membrane is initially equilibrated with an adsorbing, solute-free solution. During the first half-cycle, an adsorbing solution is fed from the left and $A$ adsorbs. Since the stroke volume is less than the membrane void volume, nonadsorbing solutes remain within the membrane; only small amounts are transported, by dispersion, to the right of the membrane. In the second half of the cycle, a solute-free desorbing solution is fed into the membrane. This solution desorbs $A$ and forms a peak of $A$ around the desorbent front. At the end of this first cycle, almost all of $B$ fed into the membrane in the first half-cycle is transported back to the left of the membrane while the adsorbed $A$ remains in the membrane. During the first half of the second cycle, some readsorption of the desorbed peak takes place, but a fraction of the desorbed peak $(A)$ is preferentially transported to the right.

From Figures 2 and 3 it is clear that rejection is similar to a noncyclic adsorption process in which adsorption and desorption occur in two distinct steps. In contrast, Figures 4 and 5 show that preferential transport is a unique process because the adsorption and desorption steps cannot be clearly distinguished. For example, during the first half-cycle, a solute wave moves under adsorbing conditions, while a desorbed solute wave concomitantly travels across the mem- brane. Also, preferential transport in adsorptive membranes is similar to facilitated transport in liquid membranes because, in both cases, a solute that interacts with the membrane is preferentially transported across the membrane. However, the two mechanisms are completely different because preferential transport does not rely on the diffusion of solute-carrier complexes in the membrane.

\section{Controlling solute movement}

The only difference between the systems in Figures 3 and 5 is the ratio of the stroke volume to the membrane void volume $\left(V_{s} / V_{m}\right)$. This ratio can therefore be used to control the direction of transport of the adsorbing solute. The effect of $V_{s} / V_{m}$, both theoretical and experimental, on the solute fluxes obtained at coherence is shown in Figure 6. These fluxes are presented in terms of dedimensionalized fractional fluxes $\left(F_{i}\right)$, where $F_{i}$ is defined as the ratio of the amount of solute $i$ transported across the membrane in a cycle to the amount of solute $i$ fed to the membrane in the cycle. Note that coherence was verified experimentally: at coherence, the amount of solute collected in the two effluent beakers in a cycle was equal to $100 \pm 3 \%$ of the solute fed in that cycle $(100 \pm 3 \%$ is the average and the standard deviation of all the experiments shown in Figure 6). 


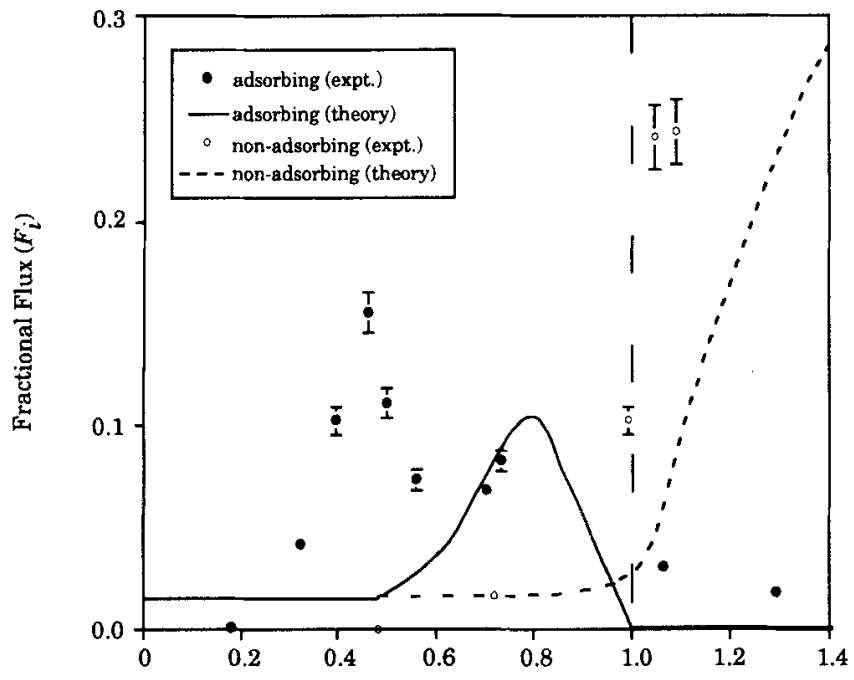

Stroke Volume/Membrane Void Volume $\left(V_{s} / V_{m}\right)$

Figure 6. Effect of stroke volume on solute fluxes.

For $V_{s} / V_{m}$ greater than 1 , the nonadsorbing solute flux is greater than the adsorbing solute flux (rejection), while for $V_{s} / V_{m}$ less than 1 the adsorbing solute flux is greater than the nonadsorbing solute flux (preferential transport). Discussion of experimental error can be found in the section called "Controlling solute movement." All parameters are given in Table 2. Note that horizontal error bars are not included because the complexity of the error propagation of this variable is difficult to calculate that is, small errors in $V_{s, a}$ and $V_{s, d}$ can lead to significant errors in $F_{i}$, see Figure 13)

Figure 6 shows that for $V_{s} / V_{m}$ greater than 1 the adsorbing solute fractional flux is close to 0 because the adsorbing solute is retained by the membrane (rejection mode). Alternatively, for the same values of $V_{s} / V_{m}\left(V_{s} / V_{m}>1\right)$, the nonadsorbing solute flux increases dramatically. This dramatic increase of nonadsorbing solute flux is because, for $V_{s} / V_{m}>1$, the nonadsorbing solute is convectively transported across the membrane in the forward stroke. Note that for $V_{s} / V_{m}$ greater than 3 for these particular experiments, the adsorbing solute flux starts to increase (data not shown) because the membrane capacity is exceeded.

Figure 6 also shows that when $V_{s} / V_{m}$ is between 0.5 and 1.0 , the adsorbing solute is preferentially transported through the membrane. The theoretical curve for the adsorbing solute fractional flux has its maximum value at $V_{s} / V_{m}$ equal to 0.8 . At this point the adsorbing solute flux is more than 5 times higher than the nonadsorbing solute flux. Note that for $V_{s} / V_{m}$ less than 1 , the nonadsorbing solute flux is low because it is transported by dispersion only. For $V_{s} / V_{m}$ less than 0.5 , the adsorbing solute flux is equal to the nonadsorbing solute flux because the desorbent wave is not able to reach and desorb the adsorbed solute. For $V_{s} / V_{m}$ less than 0.5 , the adsorbing solute is transported across the membrane only by dispersion, the same mechanism responsible for nonadsorbing solute transport.

The theoretical curve was based on predictions using independently determined parameters given in Table 2 . The void volume of the membrane was found to be $1.16 \mathrm{~mL}$, obtained by multiplying the porosity $(0.825)$ and the bed volume (1.4 $\mathrm{mL}$ ). The Peclet number, obtained using a correlation for the plate height by Dolan (1987) and $P e$ correlation by Giddings (1965), was determined as 2,100. This estimated Peclet number is only $50 \%$ higher than that measured by breakthrough studies through similar membrane cartridges (Gerstner et al., 1992). The Langmuir adsorption isotherm parameters, obtained by measuring dynamic capacities at various salt concentrations, were fitted to the following exponential functions:

$$
\begin{aligned}
& K_{m}=69.8 * 0.0024 *(I)^{-1.55} \mathrm{~mL} / \mathrm{mg} \\
& Q_{m}=18.2 * 0.0046 *(I)^{-1.38} \mathrm{mg} / \mathrm{mL},
\end{aligned}
$$

where $I$ is the sum of the buffer concentration and the salt concentration. Dynamic capacities rather than static or total capacities were used because, for typical flow rates, the cyclical nature of recuperative parametric pumping prevents the total capacity of the membrane from being used. Figure 7 shows that the exponential fits, Eqs. 19 and 20, represent the experimental data fairly well. Note that the fits shown in Figure 7 are over the entire range of ionic strengths and not fits at individual ionic strengths. Similar exponential relationships have been used to model the adsorption of proteins under varying salt concentrations (Whitley et al., 1991).

The theoretical predictions were compared with results obtained from recuperative parametric pumping experiments. Figure 6 shows that the experimental results follow the same trend as the theoretical predictions. Both experimental and

Table 2. Parameters Used in the Simulations*

Estimated parameters
Membrane void volume
Peclet number
Equilibrium constant at $0 \mathrm{M} \mathrm{KCI}$
Membrane saturation capacity at $0 \mathrm{M} \mathrm{KCl}$
Function relating equilibrium constant to ionic strength
Function relating membrane capacity to ionic strength
Dimensionless spatial step increment
Dimensionless temporal step increment
Experimental parameters
Lysozyme concentration in feed
lonic strength (KCl+buffer concentration)
Ratio of stroke volume to membrane void volume
Ratio of adsorbing stroke volume to desorbing stroke volume

$V_{m}=1.16 \mathrm{~mL}$

$P e=2,100$

$K_{m}^{\max }=69.8 \mathrm{~mL} / \mathrm{mg}$

$Q_{m}^{\max }=18.2 \mathrm{mg} / \mathrm{mL}$

$f=0.0024 * I^{-1.55}$

$g=0.004,6 * I^{-1.38}$

$\Delta z=0.005$

$\Delta \tau=0.002$

$C_{0}=4.0 \mathrm{mg} / \mathrm{mL}$

$I=0.37 \mathrm{M}$

$V_{s} / V_{m}\left(\right.$ or $\left.V_{s, a} / V_{m}\right)=0.8$

Ratio of stroke volume to membrane void volume
Ratio of adsorbing stroke volume to desorbing stroke volume

$V_{s, a}^{s} / V_{s, d}=1.02$

*Unless otherwise indicated, these parameters were used in all the simulations. 


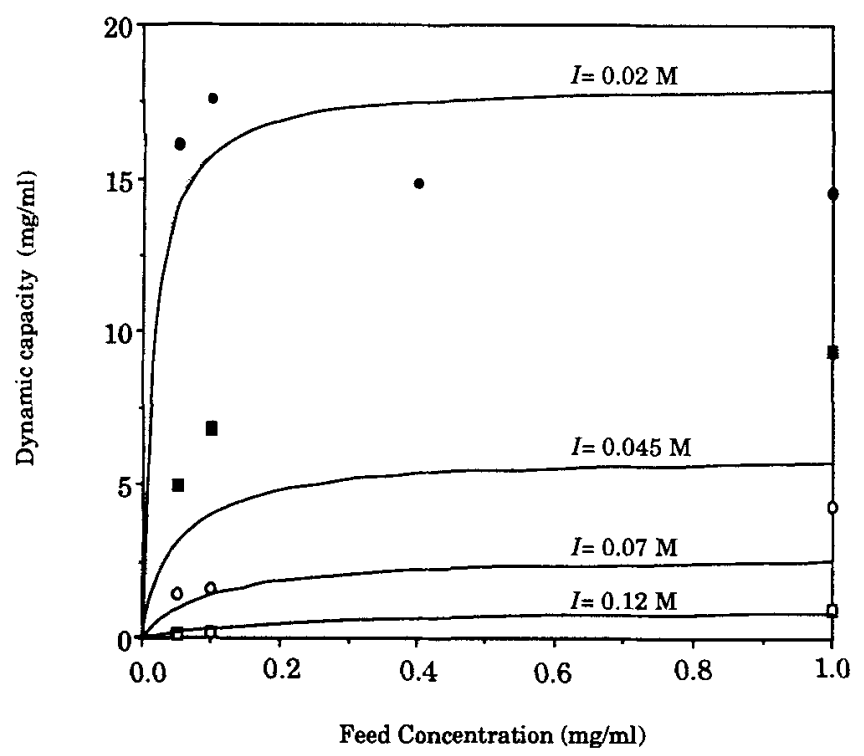

Figure 7. Adsorption isotherms at various ionic strengths.

The adsorption isotherm proposed by Antia and Horvath (1989), which is given by Eqs. 3, 4a, and $4 b$, represents the experimental data fairly well over the entire range of ionic strengths. Note that, if a Langmuir isotherm was fit to data for one ionic strength, the theory and experiments agree quite well (not shown).

theoretical results show an adsorbing solute flux maxima at values of $V_{s} / V_{m}$ less than 1 , and a dramatic increase of nonadsorbing solute flux for $V_{s} / V_{m}$ close to 1 . Scatter in the experimental data due to fractional flux measurements is minimal. The figure also shows that the experimental data for both adsorbing and nonadsorbing solutes lie to the left of the theoretical curves. Fits in which the membrane void volume is $1.0 \mathrm{~mL}$ rather than $1.16 \mathrm{~mL}$ (all other parameters remain the same) agree very well with the nonadsorbing solute experimental data and lie closer to the adsorbing solute experimental data. The better fits obtained with a lower membrane void volume could be simply a result of an error in the membrane void volume measurement or could be due to a lower effective membrane void volume during the cyclic process.

The discrepancy between experimental and theoretical adsorbing solute fluxes may be due to parameter sensitivity and model simplicity. The use of dynamic capacity is a conservative estimate of the capacity available during recuperative parametric pumping and will lead to the underprediction of experimental fluxes. In addition, adsorbing solute fluxes may change due to mixing effects in the distributor of the membrane cartridge (Roper and Lightfoot, 1993), an effect that is not accounted for in our model. Later, it is shown that dispersion not only influences the adsorbing solute flux, but also affects the location of the adsorbing solute flux curve shown in Figure 6. Finally, the model does not account for induced salt gradients that will form due to protein adsorption. However, calculations based on the protein charge, isotherm parameters, and protein and buffer concentrations show that the induced salt gradient should not significantly affect the adsorbing solute fluxes (calculations not shown). In summary, although the quantitative agreement between model and experimental results needs further improvement, the model does predict the phenomenon of preferential transport and can be used to estimate selectives that would be obtained experimentally.

\section{Methods for obtaining preferential transport}

The effect of $V_{s} / V_{m}$ (the displacement volume to membrane void volume ratio) shown in Figure 6 is similar to a plot presented by Sweed and Rigaudeau (1975), but the origin of separation in the two studies is completely different. The origin of separation of preferential transport by Sweed and Rigaudeau (1975) was attributed to mass-transfer effects. But in our study, mass-transfer effects are negligible. Therefore, since preferential transport can arise from very different experimental conditions, a complete understanding of the cause of preferential transport must be known before experimental conditions to optimize preferential transport can be predicted.

Preferential transport is a result of the adsorbing solute crossing over from the adsorbing phase to the desorbing phase within a medium. The medium could be an adsorptive membrane, such as that used in this study, or a packed bed. This crossing over of the adsorbing solute can be seen in Figures $5 \mathrm{c}$ and $5 \mathrm{~d}$. In Figure $5 \mathrm{c}$, the adsorbed solute is only present in the adsorbing phase; however, in Figure $5 \mathrm{~d}$ desorbed solute is present both ahead of and behind the desorbing wave. Thus, a fraction of the solute has crossed over from the adsorbing phase to the desorbing phase within the medium and will be transported across the medium in the next cycle (Figures $5 \mathrm{e}-5 \mathrm{i}$ ).

The fraction of solute that crosses over to the desorbing phase is a strong function of the adsorption isotherm. For example, this fraction is low for linearly sorbed solutes. Specifically, the fraction is 0 for a linearly sorbed solute under the ideal conditions of local equilibrium and no dispersion. In Figure 8, the trajectories of solvent, a linearly sorbing solute, and desorbent are shown by using characteristics (waves of constant concentration) that have slopes equal to each species' propagation velocity. The slopes of the solvent and the desorbent characteristics are the same and are equal to the interstitial fluid velocity, $u$. The characteristic slope of the adsorbing solute is given by Eq. $21\left(K_{\mathrm{eq}}=\right.$ equilibrium constant), which is obtained by simplifying Eq. 1 under the assumption of ideal conditions and linear isotherm:

$$
u_{A}=\frac{u}{1+K_{\mathrm{eq}}}
$$

Figure 8 illustrates that the solute characteristics do not intersect with the desorbent characteristics. In other words, the solute does not cross over from the adsorbing phase to the desorbing phase and preferential transport does not occur.

In contrast, preferential transport can occur for a solute whose equilibrium is described by a Langmuir isotherm (Figure 9). For Langmuir isotherms under ideal conditions, the slope of the adsorbing solute characteristic, obtained from Eqs. 1 and 3, is given by

$$
u_{A}=\frac{u}{1+\frac{1}{\epsilon} \frac{d Q_{A}}{d C_{A}}}, \quad \text { where } \quad \frac{d Q_{A}}{d C_{A}}=\frac{Q_{m} K_{m}}{\left(1+K_{m} C_{A}\right)^{2}}
$$




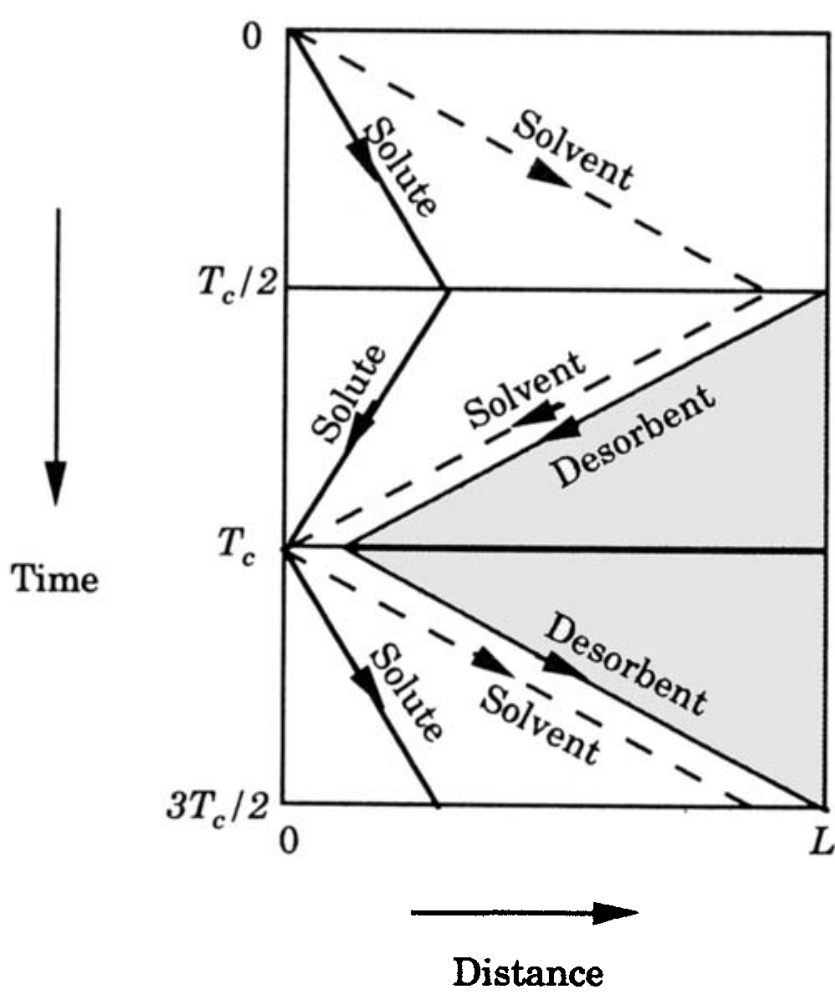

Figure 8. Time/displacement diagram for preferential transport under ideal conditions (linear isotherm).

This time /displacement diagram shows the characteristics of the solute solvent, and the desorbent. This diagram shows that in the second half of a cycle $\left(T_{c} / 2<t<T_{c}\right)$ solute and desorbent characteristics do not intersect. As a result, solutes do not cross over from the adsorbing phase to the desorbing phase and preferential transport does not occur.

Equation 22 implies that for Langmuir isotherms the slope of the adsorbing solute characteristic, or $u_{A}$, is a function of concentration. Moreover, the characteristic slope increases as concentration increases. Therefore, a low concentration characteristic propagates slower than a high concentration characteristic. These differing propagation velocities lead to a fan of characteristics (a group of characteristics emerging from a single point and diverging, or fanning out, from each other) when the desorbing solution enters the membrane (Figure 9). Note that Figure 9 shows only two characteristics, a low concentration and a high concentration, of the infinite number that constitute the fan; the complete fan of characteristics is represented by the dark shaded region. The solute that belongs to the fraction of this fan that intersects the desorbent wave crosses over from the adsorbing phase to the desorbing phase and preferential transport occurs.

Other phenomena in addition to a nonlinear isotherm can also lead to solute crossover. For example, if desorption kinetics are slow, the adsorbing solute may desorb into the desorbent phase and be preferentially transported. By a similar mechanism, mass-transfer resistances can also lead to preferential transport. All the previous packed-bed studies that have been conducted in the preferential transport regime dealt with nonlinearly sorbing solutes (Wilhelm et al., 1966; Rolke and Wilhelm, 1969; Sweed and Rigaudeau, 1975; Shaffer and

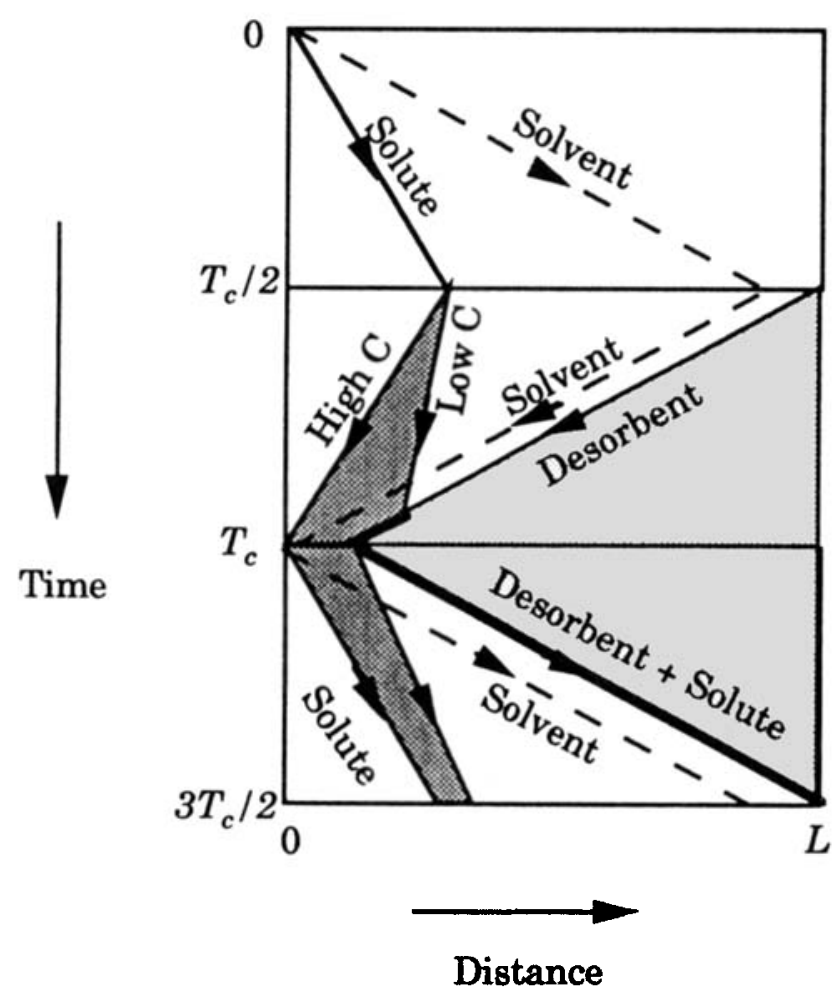

Figure 9. Time/displacement diagram for preferential transport under ideal conditions (Langmuir isotherm).

In the second half of a cycle $\left(T / 2<t<T_{c}\right)$, low concentration characteristics and the desorbent characteristic intersect and solute crossover from the adsorbing phase to the desorbing phase occurs. In the first half of the next cycie $\left(T_{c}<t<3 T_{/} / 2\right)$, the intersecting solute characteristics propagate with the desorbent wave across the membrane.

Hamrin, 1975; Chen et al., 1977). In each of these, preferential transport could be a result of a combination of nonlinear isotherm and mass-transfer effects. As mentioned earlier in the "Model Development" section, mass-transfer effects are negligible under the conditions used in this study. Therefore, in this study, preferential transport is solely due to the nonlinear isotherm.

\section{Effect of process variables in preferential transport}

The maximum theoretical limit of the adsorbing solute flux during preferential transport can easily be determined. This maximum theoretical flux occurs under the hypothetical conditions of infinite equilibrium constant and zero dispersion when the stroke volumes equal the membrane void volume. For this hypothetical case, during the adsorbing stroke, solute moves into the membrane with concentration in the pore equal to the feed concentration $\left(C_{A, 0}\right)$ and concentration on the surface equal to the saturation capacity $\left(Q_{m}^{\max }\right)$. During the desorbing stroke, all the solute present in the pore is rejected while the solute on the surface desorbs and moves behind the desorbed wave. During the next adsorbing stroke, all the desorbed solute is preferentially transported. Hence the maximum theoretical limit of the adsorbing solute flux during preferential transport is given by $Q_{m}^{\max } /\left(\epsilon C_{A, 0}+Q_{m}^{\max }\right)$. 


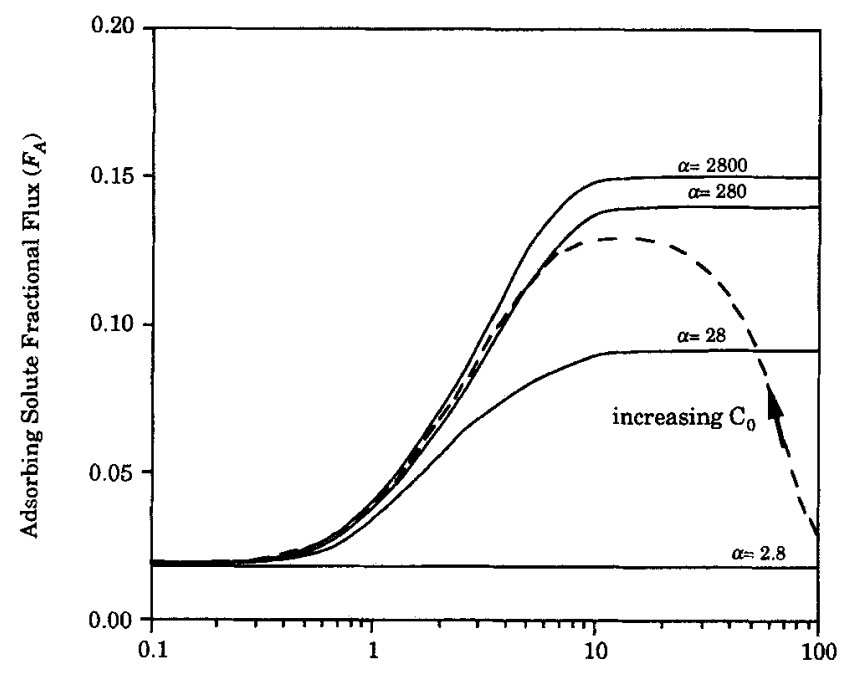

Dimensionless Membrane Capacity ( $\delta$ )

Figure 10. Effect of isotherm parameters on adsorbing solute fluxes during preferential transport.

At low $\alpha$ (isotherm linearity parameter), the Langmuir isotherm is in its linear regime and there is no preferential transport. At high $\alpha$, preferential transport occurs as Iong as $\delta$ (dimensionless membrane capacity) is also high. Since the feed concentration $\left(C_{0}\right)$ is in the numerator of $\alpha$ and the denominator of $\delta$, the adsorbing solute flux first increases and then decreases as concentration is increased (the dotted line on the figure is produced by varying concentration only). All parameters not listed are given in Table 2. Note that for the parameters given in Table 2, $\alpha=280$ and $\delta=5.6$.

When the saturation capacity ( $Q_{m}^{\max }$ ) is much higher than the feed concentration $\left(C_{A, 0}\right)$, the maximum possible flux approaches 1.

The theoretical case just described will lead to an ideal separation in which the adsorbing solute flux is 1 and the nonadsorbing solute flux is 0 . In real systems, however, the fluxes will be significantly different from these maximums. For example, in our experimental system we have obtained an adsorbing solute flux on the order of 0.1 and a nonadsorbing solute flux on the order of 0.01 . To design efficient separation systems, the influence of each process variable on solute fluxes must be known.

Adsorption Isotherm. Preferential transport can be enhanced by using an adsorbing membrane with higher equilibrium constants and higher adsorbent capacities (Figure 10). Figure 10 shows that increase in the two isotherm parameters, the isotherm linearity parameter given by $\alpha$ (the product of equilibrium constant and feed concentration) and the dimensionless membrane capacity parameter given by $\delta$ (ratio of adsorbent capacity to feed concentration), leads to increase in the adsorbing solute flux. In addition, the figure shows that above a threshold value of $\alpha \approx 10^{3}$ and $\delta \approx 10$, there is little increase in adsorbing solute fluxes. Most chromatographic media, including our experimental system, are close to these threshold values of $\alpha$ and $\delta$ and further increase will not improve the flux.

Figure 10 also shows that as the feed concentration increases, adsorbing solute flux first increases and then decreases. At low concentrations $\left(C_{A, 0} \ll 1 / K_{m}^{\max }\right)$, Langmuir isotherms become linear, and the adsorbing solute flux is therefore low. At high concentrations $\left(\epsilon C_{A, 0} \gg Q_{m}^{\max }\right)$ the slope of the isotherm $\left(d Q_{A} / d C_{A}\right.$ given by Eq. 22$)$ is zero and there is no preferential transport. This is because when the slope of the isotherm is zero, the propagation velocities of the shock wave and the diffuse wave are equal (the shock wave is the adsorbing solute wave formed during the adsorbing stroke, while the diffuse wave is the adsorbing solute wave formed during the desorbing stroke). From Figure 10 one can calculate that for our experimental system the maximum adsorbing solute flux occurs in the range of $C_{0}=1-5 \mathrm{mg} / \mathrm{mL}$; the experimental value $(4 \mathrm{mg} / \mathrm{mL})$ lies in this range and no further increase in flux for our system can be expected by changing the feed concentration.

Dispersion. The adsorbing solute fluxes shown in Figure 10 are much lower than the theoretical maximum flux. As mentioned earlier, the theoretical maximum flux can be obtained under the hypothetical conditions of infinite equilibrium constant and zero dispersion. For example, for our experimental system the theoretical maximum flux is $0.85\left(Q_{m}^{\max }\right.$ $=18.2 \mathrm{mg} / \mathrm{mL}, \epsilon=0.825, C_{A, 0}=4 \mathrm{mg} / \mathrm{mL}$, and the theoretical maximum flux equals $Q_{m}^{\max } /\left(\epsilon C_{A, 0}+Q_{m}^{\max }\right)$. Figure 10 shows that for our experimental conditions, even if the equilibrium constant approaches infinity, flux approaches a value lower than 0.2 . This drastic lowering of flux from 0.85 to 0.2 is mainly due to dispersion.

Figure 11 shows that high Peclet numbers (low dispersion) promote preferential transport, while low Peclet numbers (high dispersion) prevent preferential transport. The figure also shows that both regimes of $P e$ lead to a separation. For low dispersion $\left(P e>10^{3}\right)$ adsorbing solute flux increases dramatically while nonadsorbing solute flux remains constant. This suggests that media leading to low dispersion are re-

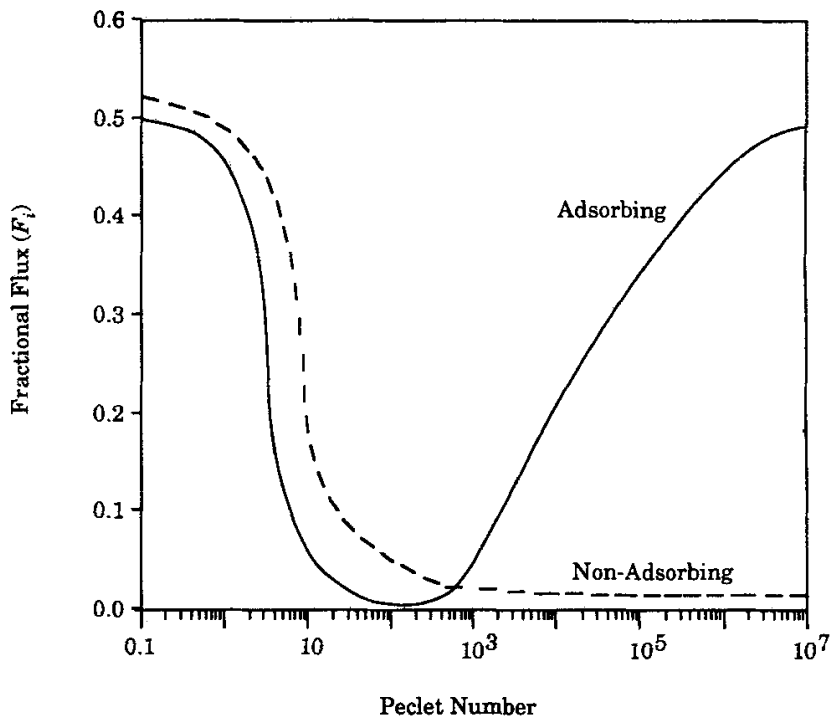

Figure 11. Effect of dispersion on solute fluxes during preferential transport.

For low dispersion $\left(\mathrm{Pe}>10^{3}\right)$, the adsorbing solute is pref erentially transported while, for high dispersion $\left(P e<10^{3}\right)$ preferential transport is eliminated by axial fluid mixing. All parameters are given in Table 2 . Also, $P e$ and the corresponding finite difference methods used to generate this figure were, for $P e<10^{3}$, the implicit state variable method $(\Delta z=0.005, \Delta \tau=0.005)$, for $P e>10^{3}$, the Dufort Frankel method $(\Delta z=0.005, \Delta \tau=0.002)$, and for $P e=\nsim$, the backward explicit method $(\Delta z=0.005, \Delta \tau=0.005)$. 


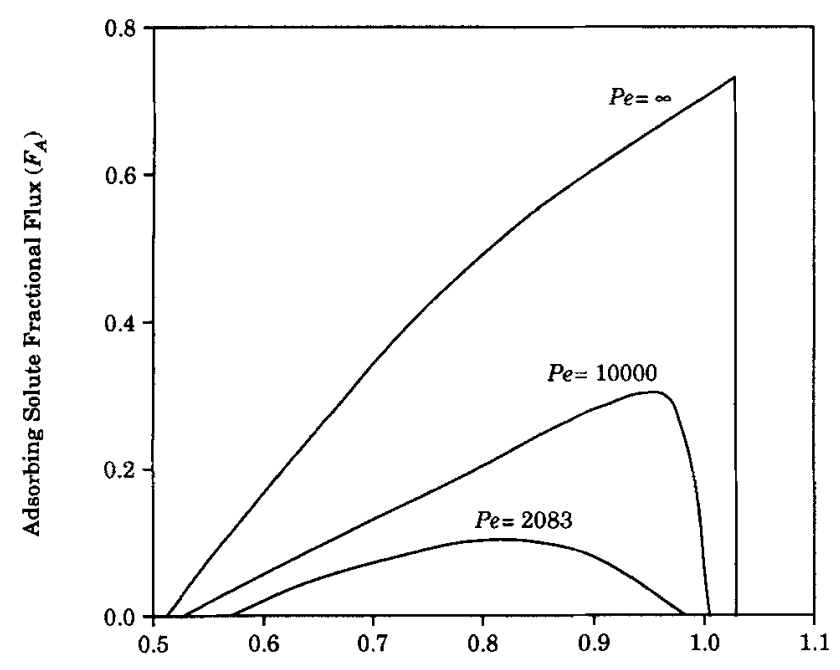

Displacement Volume/Membrane Void Volume $\left(V_{s} / V_{m}\right)$

Figure 12. Effect of dispersion on adsorbing solute fluxes during preferential transport.

The value of $V_{s} / V_{m}$ at which the maximum adsorbing solute flux is obtained is higher for higher $P e$ values. For $P e=x$, the adsorbing solute flux is 0.73 , which is $86 \%$ of the theoretical maximum flux if all other parameters remain constant, indicating the importance of dispersion. The sharp drop off at $V_{s} / V_{m}=1.02$ is due to desorbent breakthrough at this value $\left(V_{s} / V_{m}\right.$ or $V_{s, a} / V_{m}=1.02, V_{s, d} / V_{s, d}=$ 1.02 , therefore, $\left.V_{s, d} / V_{m}=1\right)$. All parameters are given in Table 2 .

quired for improved separations using preferential transport. Packed beds have $P e$ in the range $3 \times 10^{4}$ to $10^{6}$ (Phillips et al., 1988) and may lead to highly selective preferential transport separations. However, packed beds are typically masstransfer limited, and experimentally obtained selectivities could be substantially lower. A comparison between membranes and packed beds could be done if mass-transfer terms are added to the model presented in this study. Finally, Figure 11 shows that for high dispersion $\left(P e<10^{3}\right)$, the adsorbing solute flux is lower than the nonadsorbing solute flux. In this regime, the desorbent wave is so dispersed that it carries the desorbed solute to the left of the membrane, leading to rejection (refer to Figure 5f).

Figure 12 shows that the maximum adsorbing solute flux for no dispersion (but all other parameters remaining the same) is 0.73 . This value is $86 \%$ of the theoretical maximum flux for this experimental system (0.85), suggesting the importance of using a low dispersion medium. The figure also shows that dispersion leads to a shift in the optimum value of $V_{s} / V_{m}$ (the displacement volume to membrane void volume ratio). For a medium with no dispersion, the optimum value of $V_{s} / V_{m}$ is close to 1. As dispersion increases (or as $P e$ decreases), this optimum value shifts to values lower than 1 .

Figures 11 and 12 show that separations using preferential transport will significantly improve if a membrane with low dispersion $\left(P e>10^{4}\right)$ is used. We are not aware of such a low dispersion membrane; however, a membrane having uniform cylindrical pores will lead to low $P e$. Pe can be calculated very accurately for dilute solutions flowing in a cylinder under laminar flow using the relationship derived by Taylor (1953). Using Taylor's formula and the conditions used in this study, $P e$ for a membrane with uniform cylindrical pores is about $2 \times 10^{6}\left(P e=48 D L / r^{2} u, u=0.004 \mathrm{~cm} / \mathrm{s}, L=0.5 \mathrm{~cm}\right.$, $\left.r=0.6 \mu \mathrm{m}, D=1.1 \times 10^{-6} \mathrm{~cm}^{2} / \mathrm{s}\right)$. The challenge in making such a low dispersion membrane is to obtain uniform cylindrical pores.

Asymmetric Strokes. The nonadsorbing solute fluxes shown in Figure 11 are higher than the theoretical minimum flux, which we showed earlier is equal to 0 . The flux is nonzero because the adsorbing stroke volume $\left(V_{s, a}\right)$ is greater than the desorbing stroke volume $\left(V_{s, d}\right)\left(V_{s, a} / V_{s, d}=1.02\right.$ for the plot $)$. Such an operation in which the adsorbing stroke volume is not equal to the desorbing stroke volume is referred to as an operation with asymmetric strokes. The effect of asymmetric strokes on solute fluxes has been studied using the ratio of the adsorbing stroke volume to the desorbing stroke volume, $V_{s, a} / V_{s, d}$.

Using asymmetric strokes in which $V_{s, a} / V_{s, d}>1$ leads to enhanced adsorbing solute fluxes, but usually lowers the selectivity that can be obtained in a given separation due to the increased nonadsorbing solute fluxes (Figure 13). Figure 13 shows that the experimental results follow the same trend as the theoretical results for a variety of $V_{s, a} / V_{s, d}$ values. The discrepancy between experimental and theoretical adsorbing solute flux values is probably due to reasons mentioned earlier in the discussion of Figure 6. Scatter in the experimental data due to measurements of fractional flux is low, while scatter due to variations in $V_{s, a} / V_{s, d}$ (the ratio of the pump flow rates) is about $5 \%$. These variations in $V_{s, a} / V_{s, d}$ probably do not affect fractional flux measurements because the fractional flux measurements are made from an average of multiple $(\sim 5)$ cycles.

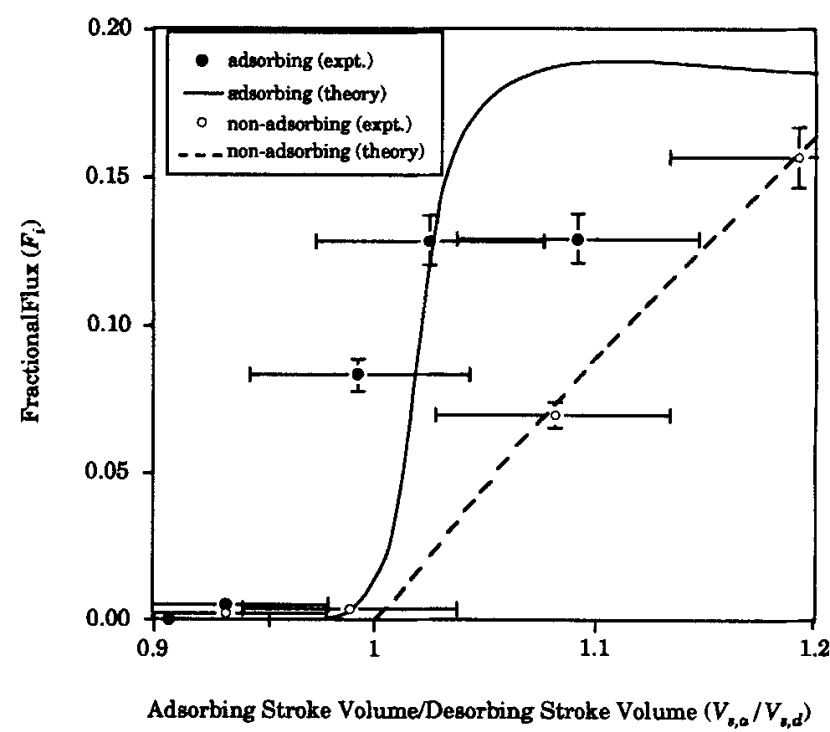

Figure 13. Effect of asymmetric strokes on solute fluxes during preferential transport.

Increasing the ratio of adsorbing stroke volume to the desorbing stroke volume $\left(V_{s, a} / V_{s, d}\right)$ leads to increase in fluxes of both adsorbing and nonadsorbing solutes. Moreover, as $V_{s, a} / V_{s, d}$ is increased, the ratio of adsorbing to nonadsorbing solute flux decreases for all values of $V_{s, a} / V_{s, d}$. Therefore, the optimum $V_{s, d} / V_{s, d}$ will be a tradeoff between desired flux and selectivity. Discussion of experimental error can be found in the section called "Effect of asymmetric strokes." All parameters are given in Table 2 . 
Figure 13 also shows that the adsorbing solute flux increases dramatically when $V_{s, a} / V_{s, d}$ is increased from 1.0 to 1.05 , while for values of $V_{s, a} / V_{s, d}$ greater than 1.05 , the adsorbing solute flux is almost constant. This dependence on $V_{s, a} / V_{s, d}$ can be qualitatively understood with the help of Figure 5, which was constructed for $V_{s, a} / V_{s, d}=1.02$. When $V_{s, a} / V_{s, d}$ is less than 0.95 , the adsorbing solute wave at the end of the first half of cycle 2 would look like Figure 5h, in which none of the desorbed peak is transported through the membrane. Thus, for this case, the adsorbing solute flux would be low. As we increase $V_{s, a} / V_{s, d}$ from 0.95 to 1.05 , the adsorbing solute wave at the end of the first half of cycle 2 would look like Figure 5i. For $V_{s, a} / V_{s, d}$ from 0.95 to 1.05 , the fraction of desorbed peak transported increases dramatically and accounts for the increase of the adsorbing solute flux in Figure 13. For $V_{s, a} / V_{s, d}$ greater than 1.05, all of the peak of Figure $5 \mathrm{i}$ is transported to the right, and further increase in $V_{s, a} / V_{s, d}$ does not lead to an increase in adsorbing solute flux. For $V_{s, a} / V_{s, d}$ greater than 1.3 , the adsorbing solute begins to break through and the adsorbing solute flux is approximately equal to the nonadsorbing solute flux.

Figure 13 shows that as $V_{s, a} / V_{s, d}$ increases, selectivity decreases due to the increase of nonadsorbing solute flux. This decrease of selectivity with increasing $V_{s, a} / V_{s, d}$ was verified by experiments in which a multicomponent feed consisting of lysozyme (adsorbing) and myoglobin (nonadsorbing) was used. For the ratios of 1.00, 1.03, and 1.04, selectivities obtained at coherence (flux of lysozyme/flux of myoglobin) were 4.41, 3.71 , and 2.00 , respectively; thus increase in $V_{s, a} / V_{s, d}$ led to decrease in selectivity. However, increases in $V_{s, a} / V_{s, d}$ from below to above 1 resulted in more than an order of magnitude increase in the flux of the adsorbing solute. Note that this sensitivity to $V_{s, a} / V_{s, d}$, while providing a means to increase adsorbing solute flux, may lead to control problems during actual operation.

In order to increase selectivity, we would like to operate at values of $V_{s, a} / V_{s, d}$ less than or equal to 1.0 and use a suitable desorbent, specifically, a desorbent that leads to isotherm functions $f$ and $g$ (Eq. 4) that are closer to step functions rather than exponential functions. These relationships (holding all other values in Table 2 constant) are given by

$$
\begin{aligned}
& K_{m}=69.8 \mathrm{~mL} / \mathrm{mg} \quad \text { for }[I]>0.185 \mathrm{M} \\
& =0.01 \quad \text { for }[I] \leq 0.185 \mathrm{M}
\end{aligned}
$$

and

$$
\begin{aligned}
Q_{m} & =18.2 \mathrm{mg} / \mathrm{mL} & & \text { for }[I]>0.185 \mathrm{M} \\
& =0.01 & & \text { for }[I] \leq 0.185 \mathrm{M} .
\end{aligned}
$$

Figure 14 compares the fluxes obtained by the hypothetical step desorbent with the exponential desorbent. The figure shows that for the step desorbent, high adsorbing solute fluxes can be obtained for $V_{s, a} / V_{s, d}$ less than or equal to 1.0. For instance, at $V_{s, a} / V_{s, d}$ equal to 1.0 the adsorbing and nonadsorbing solute fluxes are 0.18 and 0.0034 , respectively, for the step. This increase in selectivity (53 as opposed to 5.5 ) can be understood with the help of Figure $5 \mathrm{f}$. In the figure, which is for an exponential desorbent (modeled by Eqs. 19 and 20), most of the desorbed peak lies ahead of the desorbent front.

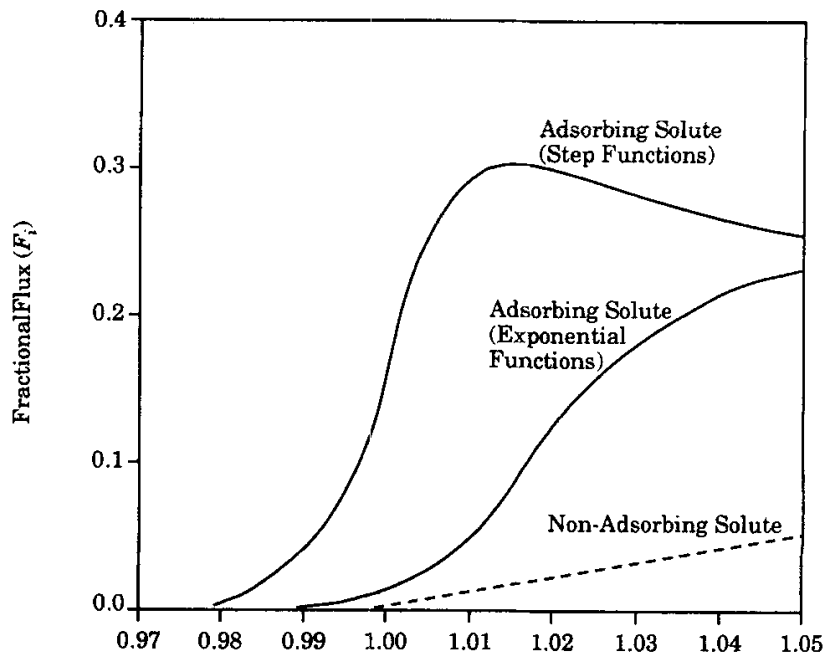

Adsorbing Stroke Volume/Desorbing Stroke Volume $\left(V_{s, a} / V_{s, d}\right)$

Figure 14. Effect of desorbent on adsorbing solute fluxes during preferential transport.

For a desorbent that leads to step isotherm functions rather than exponent isotherm functions, high adsorbing solute fluxes are obtained at values of $V_{s, a}, V_{s, d}$ less than or equal to 1.0. Such a desorbent leads to high flux and selectivity. All parameters are given in Table 2 .

This desorbed peak partially readsorbs in the next half cycle (Figure $5 g-5 i$ ), leading to low fluxes. For a step desorbent (modeled by Eqs. 23 and 24), the desorbed peak is mostly within the desorbent front (simulation result not shown). For this case, the desorbed peak does not readsórb in the next half-cycle and high fluxes are obtained.

\section{Continuous rejection and preferential transport}

The adsorbing and nonadsorbing solute fluxes discussed thus far were determined for the semicontinuous system shown in Figure 1. With careful analysis, these fluxes can be used to design a wide variety of continuous and semicontinuous separation systems. One possible continuous configuration is shown in Figure 15; the continuous contactor consists of two stirred reservoirs separated by an adsorptive membrane stack. The left reservoir contains an adsorbing solution, while the right reservoir contains a nonadsorbing solution. Into the left reservoir flows a desorbing solution containing an adsorbing solute $A$ and a nonadsorbing solute $B$, whereas into the right reservoir flows a solute-free desorbing solution. Exchange of solutes between the two reservoirs takes place due to an oscillatory flow in the membrane. The oscillatory flow exposes the membrane to alternately adsorbing and desorbing conditions, leading to recuperative parametric pumping as described earlier.

To evaluate the performance of rejection and preferential transport in this continuous contactor, the following steadystate mass balances are solved:

$$
\begin{gathered}
C_{i, 0} q_{0}-C_{i, L} q_{L}-F_{i} C_{i, L} \nu V_{s, a}=0 \\
C_{i, D} q_{D}-C_{i, R} q_{R}+F_{i} C_{i, L} \nu V_{s, a}=0
\end{gathered}
$$




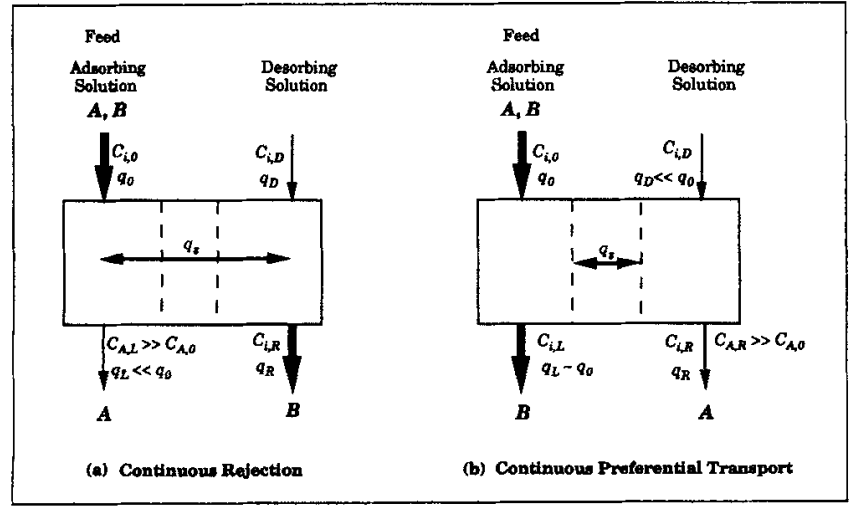

Figure 15. Rejection and preferential transport in continuous contactors.

(a) During continuous rejection, the adsorbing solute $A$ is concentrated in the left reservoir, while the nonadsorbing solute $B$ is transported across the membrane to the right reservoir. (b) During continuous preferential transport, the nonadsorbing solute $B$ is retained in the left reservoir, while the adsorbing solute $A$ is transported across the membrane to the right reservoir.

where $q_{0}, q_{D}, q_{L}$, and $q_{R}$ are the flow rates of feed, desorbent, left product, and right product, respectively; $v$ is oscillation frequency; $V_{s, a}$ is the volume displaced in the adsorbing stroke; $C_{i, l}, C_{i, D}, C_{i, l}$, and $C_{i, R}$ are the concentrations of solute $i$ in the feed, desorbent, left product, and right product, respectively; and $F_{i}$ is the fractional flux of solute $i$. In Eqs. 25 and 26, we have assumed that $F_{i}$ is identical to that calculated from the earlier semicontinuous analysis. Although $F_{i}$ must be corrected for continuous operation (for example, the desorbing solution is no longer free of solute, the feed is no longer free of desorbent), we will show that these corrections are negligible and that the values of $F_{i}$ shown earlier in this article can be used for a first-order design.

Continuous rejection (Figure 15a) can lead to high selectivity and recovery when highly asymmetric oscillations are used $\left(V_{s, a} / V_{s, d} \gg 1\right)$. These asymmetric oscillations can be produced by using a feed flow $\left(q_{0}\right)$ higher than the left product flow $\left(q_{L}\right)$ so that a net transmembrane convective flow is superimposed on the oscillatory flow. Figure 16 shows that as $q_{0} / q_{L}$ increases, selectivity increases while recovery decreases. For Figure 16, the values of $V_{s, a} / V_{s, d}$ and the adsorbing solute flux were fixed at 50 and 0.01 , respectively, based on experimental results $\left(C_{0}=0.1 \mathrm{mg} / \mathrm{mL}, I=0.37 \mathrm{M}\right)$. Note that for the results shown in Figure 16, the nonzero solute concentrations in the desorbent reservoir does not affect the flux term because of the highly asymmetric strokes used $\left(V_{s, a} / V_{s, d}=50\right)$. This highly asymmetric stroke ratio also prevents desorbent from accumulating in the feed side (for $q_{0} / q$ $=10$, nonadsorbing solute backflux is only $2 \%$ of forward flux and the desorbent concentration in the left reservoir is only $1 \%$ of that in the right reservoir). Figure 16 shows that continuous rejection can be used for high selectivity and high recovery operations. (For $q_{0} / q_{L}=10$, selectivity $=9$ and recovery $=91 \%$.)

Continuous preferential transport (Figure 15b) can also lead to high selectivity and recovery when the ratio of the oscillatory flow $\left(q_{s}\right)$ to feed flow $\left(q_{0}\right)$ is optimized. Figure 17

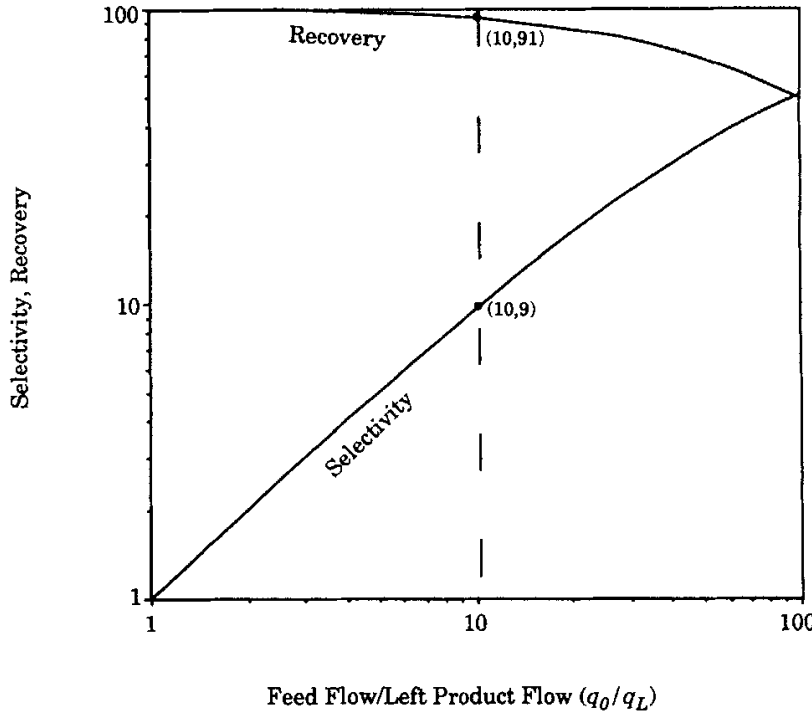

Figure 16. Performance of continuous rejection.

During continuous rejection, as the ratio of feed flow to the left product flow $\left(q_{0} / q_{L}\right)$ increases, selectivity increases while recovery decreases. High selectivity and recovery can be obtained $\left(q_{0} / q_{L}=10\right.$, selectivity $=9$, and recovery $=91 \%)$. For this figure, $F_{A}=0.01$ and $V_{s . d} / V_{s, d}=$ 50

shows that as the ratio of oscillatory flow to the feed flow increases, selectivity decreases while recovery increases. For Figure 17, three values of adsorbing solute flux were chosen, $0.1,0.5$, and 0.9 . Also, for this figure, the nonadsorbing solute flux $F_{B}$ is fixed at a typical value of $0.01\left(F_{B}=0.01\right.$, for $P e>$

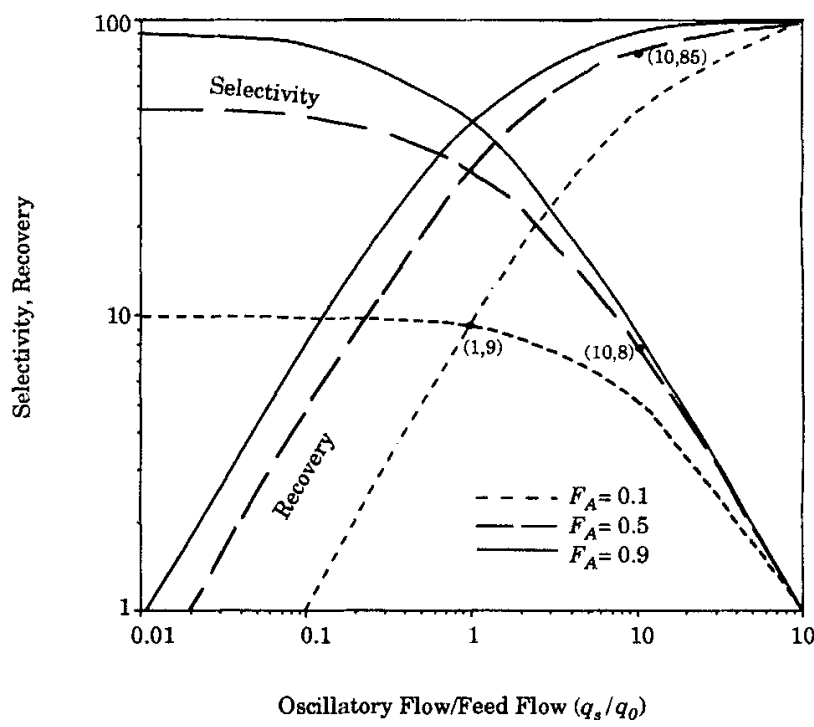

Figure 17. Performance of continuous preferential transport.

During continuous preferential transport, as the ratio of oscillatory flow to the feed flow $\left(q_{s} / q_{0}\right)$ increases, selectivity decreases while recovery increases. Two types of operation are therefore possible. If the feed stream can be recycled, high selectivities can be obtained at low $q_{s} / q_{0}$ values $\left(q_{s} / q_{0}=1\right.$ and $F_{A}=0.1$, selectivity $=9$, point $(1,9)$ on the plot). For high selectivity in single-pass operations, higher fluxes need to be obtained $\left(q, / q_{0}=10\right.$ and $F_{A}=0.5$, selectivity $=8$, recovery $=85 \%$, points $(10,8)$ and $(10,85)$ on the plot). For this figure, $F_{B}=0.01$ and $V_{s, d} / V_{s, d}=1.01$. 
500 and $V_{s, a}=V_{s, d}=1.01$; see Figures 11 and 13). Note that for the results shown in Figure 17, solute backfluxes are low $\left(F_{D} \approx 10^{-4}\right)$ because the oscillation is asymmetric with the desorbing volume lower than the adsorbing volume (see Figure 13). Figure 17 shows that continuous preferential transport can be used for selective separations in which the treated solution can be recycled. (For $q_{s} / q_{0}=1$ and $F_{A}=0.1$, selectivity $=9$.) In addition, if a higher adsorbing solute flux can be obtained, such as in a low dispersion membrane (see Figure 12), continuous preferential transport can be used for high selectivity and high recovery in single-pass operations. (For $q_{s} / q_{0}=10$ and $F_{A}=0.5$, selectivity $=8$ and recovery $=85 \%$.)

Figures 16 and 17 show that selectivities achieved in the continuous systems are not high in view of the dramatic difference between the adsorption characteristics of the solutes studied. However, the advantage of these processes is not increased selectivity. Aside from an obvious advantage of continuous operation, both these systems use oscillatory flows that alleviate concentration polarization on the membrane surface, a phenomenon that leads to reduction of solvent flux through the membrane (Belfort, 1989). Also, a unique advantage of preferential transport is that it can be applied in simultaneous size-based and adsorption-based separations. That is, the membrane can reject particles that are larger than the pore size of the membrane.

Another application in which preferential transport may be useful is when a solute is to be transported from a solution of lower concentration to a solution of higher concentration. Our simulation results indicate that the concentration of solute in the desorbing solution does not affect the adsorbing solute flux significantly. For example, for the parameters given in Table 2, a feed solution of $2 \mathrm{mg} / \mathrm{mL}$ has a flux of 0.126 when the desorbing solution is solute-free and a flux of 0.122 when the desorbing solution has a concentration of $10 \mathrm{mg} / \mathrm{mL}$. This result is expected because solute present in the desorbing solution will remain in the desorbent wave and will not affect the adsorption of solute in the adsorbing stroke. Therefore, for a continuous system, a solute can be "pumped" from a concentration of 2 to that of $10 \mathrm{mg} / \mathrm{mL}$ and be obtained in purified form.

\section{Conclusions}

Recuperative parametric pumping in adsorptive membranes can lead to two types of separations: rejection and preferential transport. Rejection uses the same mechanism of separation as a noncyclic adsorption process. In contrast, preferential transport is a unique transport mechanism. Preferential transport in adsorptive membranes is similar to facilitated transport in liquid membranes because in both cases a solute that interacts with the membrane is preferentially transported by the membrane. However, the two mechanisms are completely different because preferential transport does not rely on the diffusion of solute-carrier complexes in the membrane.

The phenomenon that leads to preferential transport is the crossover of the adsorbing solute from the adsorbing region to the desorbing region in the membrane. Preferential transport can be theoretically obtained by mass-transfer resistances, slow desorption kinetics, or nonlinear adsorption isotherms. In this study, preferential transport was obtained by a Langmuir isotherm, an isotherm that would be typical for adsorptive membranes. A mathematical model was developed that was in agreement with experimental results, and selectivities obtained, theoretically and experimentally, were on the order of 5. The results from the mathematical model suggest that in order to further improve separation by preferential transport, experiments must be conducted in low dispersion membranes $(P e>10,000)$.

Both rejection and preferential transport can lead to concentrated streams of adsorbing solutes and a number of applications can be envisioned. Continuous rejection can lead to high selectivity and recovery if highly asymmetric oscillations are used in which $V_{s, a} / V_{s, d} \gg 1$; a possible application of continuous rejection is the treatment of wastewater in which water flows through the membrane and adsorbing contaminants are retained by the membrane. Alternatively, continuous preferential transport can lead to high selectivity and recovery if the ratio of the oscillatory flow $\left(q_{s}\right)$ to feed flow $\left(q_{0}\right)$ is optimized. Preferential transport can lead to simultaneous size-based and adsorption-based separation; a possible application is the removal of a toxin that inhibits cell growth from a fermentation broth. In such a process, the toxin can be continuously removed by preferential transport through an affinity membrane specific to the toxin, while the treated feed is recycled back to the broth.

\section{Acknowledgments}

This work was partially funded by the National Science Foundation (CTS-9216078 and CTS-9096185) and the University of Michigan.

\section{Notation}

$$
\begin{aligned}
E & =\text { axial dispersion coefficient, } \mathrm{cm}^{2} / \mathrm{s} \\
l & =\text { distance in the axial direction, } \mathrm{cm} \\
t & =\text { time, } \mathrm{s}
\end{aligned}
$$

\section{Literature Cited}

Almeida, F., C. Costa, A. Rodrigues, and G. Grevillot, Physiochemical Methods for Water and Wastewater Treatment, Elsevier, Amsterdam, p. 169 (1982).

Antia, F. D., and C. Horvath, "Gradient Elution in Non-linear Preparative Liquid Chromatography," J. Chromatog., 484, 1 (1989).

Belfort, G., "Fluid Mechanics in Membrane Filtration: Recent Developments," J. Memb. Sci., 40, 123 (1989).

Brandt, S., R. A. Goffe, S. B. Kessler, J. L. O'Connor, and S. E. Zale, "Membrane-Based Affinity Technology for Commercial Scale Purifications," Bio/technology, 6, 779 (1988).

Briefs, K.-G., and M.-R. Kula, "Fast Protein Chromatography on Analytical and Preparative Scale Using Modified Microporous Membranes," Chem. Eng. Sci., 47, 141 (1992).

Brooks, C. A., and S. M. Cramer, "Steric Mass-Action Ion Exchange: Displacement Profiles and Induced Salt Gradients," AIChE J., 38(12), 1969 (1992).

Butts, T. J., N. H. Sweed, and A. A. Camero, "Batch Fractionation of Ionic Mixtures by Parametric Pumping," Ind. Eng. Chem. Fundam., 12(4), 467 (1973).

Camero, A. A., and N. H. Sweed, "Separation of Nonlinearly Sorbing Solutes by Parametric Pumping," AIChE J., 22(2), 369 (1976).

Chen, H. T., T. K. Hsieh, H. C. Lee, and F. B. Hill, "Separation of Proteins via Semicontinuous pH Parametric Pumping," AIChE J., 23(5), 695 (1977).

Chen, H. T., J. L. Rak, J. D. Stokes, and F. B. Hill, "Separations via Continuous Parametric Pumping," AIChE J., 18(2), 365 (1972). 
Chen, H. T., W. T. Yang, C. M. Wu, C. O. Kerobo, and V. Jajalla "Semicontinuous pH Parametric Pumping: Process Characteristics and Protein Separations," Sep. Sci. Tech., 16(1), 43 (1981).

Chen, H. T., W. T. Yang, U. Panchareon, and R. J. Parisi, "Separation of Proteins via Multicolumn pH Parametric Pumping," AIChE J., 26(5), 839 (1980).

Danckwerts, P. V., "Continuous Flow Systems. Distribution of Residence Times, "Chem. Eng. Sci., 2, 1 (1952).

Dolan, J. W., "Shortcuts for LC Measurements," $L C-G C, \mathbf{5}(12), 1031$ (1987).

Ferreira, L., C. Costa, and A. Rodrigues, "Adsorptive Parametric Pumping," Proc. Int. Conf. on Environmental Pollution, Vol. 2, Geneva, p. 849 (1991)

Gerstner, J. A., R. Hamilton, and S. M. Cramer, "Membrane Chromatographic Systems for High-Throughput Protein Separations," J. Chromatog., 596, 173 (1992).

Giddings, J. C., Dynamics of Chromatography I, Marcel Dekker, New York (1965).

Grevillot, G., Handbook for Heat and Mass Transfer Operations, Vol. 2, Gulf Publishing, West Orange, NJ (1986)

Guzman-Garcia, A. G., P. N. Pintauro, M. W. Verbrugge, and R. H. Hill, "Development of Space-Charge Transport Model for Ion-Exchange Membranes," AIChE J., 36(7), 1061 (1990).

Helfferich, F. G., "Multicomponent Wave Propagation: Attainment of Coherence from Arbitrary Starting Conditions," Chem. Eng, Commun., 44, 275 (1986).

Hollein, H. C., H. C. Ma, C. R. Huang, and H. T. Chen, "Parametric Pumping with $\mathrm{pH}$ and Electric Field: Protein Separations," Ind. Eng. Chem. Fundam., 21, 205 (1982).

Huang, C. R., and H. C. Hollein, Handbook of Separation Techniques for Chemical Engineers, McGraw-Hill, New York (1988).

Huang, S. Y, C. K. Lin, and L. Y. Juang, "Separation and Purification of Enzymes by Continuous pH Parametric Pumping," Biotechnol. Bioeng., 27, 1451 (1985).

Koo, Y. M., and P. C. Wankat, "Modelling of Size Exclusion Parametric Pumping," Sep. Sci. Tech., 23, 413 (1988).

Lapidus, L. and N. R. Amundson, "Mathematics of Adsorption in Beds. VI. The Effect of Longitudinal Diffusion in Ion Exchange and Chromatographic Columns," J. Phys. Chem., 56, 984 (1952).

Lapidus, L., and G. F. Pinder, Numerical Solution of Partial Differential Equations in Science and Engineering, Wiley, New York (1982).

Leighton, D. T., and M. J. McCready, "Shear Enhanced Transport in Oscillatory Liquid Membranes," AIChE J., 34(10), 1709 (1990).

McCracken, E. A., G. L. Leefe, and R. E. C. Weaver, "Transient Numerical Solution for Two-Phase Fluid-Bed Models," Chem. Eng. Prog. Symp. Ser., 66(101), 37 (1970).

Phillips, M. W., G. Subramanian, and S. M. Cramer, "Theoretical Optimization of Operating Parameters in Nonideal Chromatography," J. Chromatog., 454, 1 (1988).

Pigford, R. L., "An Equilibrium Theory of the Parametric Pump," Ind. Eng. Chem. Fundam., 8(1), 144 (1969).
Ramirez, W. F., Computational Methods for Process Simulation, Butterworth, Stoneham, MA (1989).

Rice, R. G., "Progress in Parametric Pumping," Sep. Purif. Methods, 5(1), 139 (1976).

Rolke, R. W, and R. H. Wilhelm, "Recuperative Parametric Pumping," Ind. Eng. Chem. Fund., 8(2), 235 (1969).

Roper, D. K., and E. N. Lightfoot, "Characterizing Mass Transfer in Adsorptive Bioseparations using Magnetic Resonance Imaging," Extended Abstracts, AIChE Meeting, St. Louis (1993).

Shaffer, A. G., and C. E. Hamrin, "Enzyme Separation by Parametric Pumping," AIChE J., 21(4), 782 (1975).

Suen, S.-Y., and M. R. Etzel, "A Mathematical Analysis of Affinity Membrane Bioseparations," Chem. Eng. Sci., 47, 1355 (1992).

Sweed, N.H., "Parametric Pumping and Cycling Zone Adsorption-A Critical Analysis," AIChE Symp. Ser., 80(233), 44 (1984).

Sweed, N. H., and J. M. Rigaudeau, "Adsorption and Ion Exchange," AlChE Symp. Ser., 71(152), 1(1975).

Taylor, G. I." "Dispersion of Soluble Material in Solvent Flowing Slowly Through a Tube," Proc. Roy. Soc. London, A219, 186 (1953).

Thompson, D. W., and D. Bass, "Cyclic Electrodialysis: Experimental Results in a Closed System," Can. J. Chem. Eng., 52, 345 (1974).

Wankat, P. C., "Continuous Recuperative Mode Parametric Pumping," Chem. Eng. Sci., 33, 723 (1978).

Wankat, P. C., Percolation Processes: Theory and Application, Sijthoff and Noordhoff, Alphen aan den Rijn, The Netherlands (1981).

Wankat, P. C., Large-Scale Adsorption and Chromatography, CRC Press, Boca Raton, FL (1986).

Wankat, P. C., "Liquid-Liquid Extraction Parametric Pumping," Ind. Eng. Chem. Fundam., 12(3), 372 (1973).

Weinbrenner, W. F., and M. R. Etzel, "Competitive Adsorption of $\alpha$-lactalbumin and Bovine Serum Albumin to a Sulphopropyl Ion Exchange Membrane," J. Chromatog., 662, 414 (1994).

Whitley, R. D., J. A. Berninger, N. Rouhana, and N.-H. L. Wang, "Nonlinear Gradient Isotherm Parameter Estimation for Proteins with Consideration of Salt Competition and Multiple Forms," Biotechnol. Prog., 7, 544 (1991).

Wilhelm, R. H., A. W. Rice, and A. R. Bendelius, "Parametric Pumping: A Dynamic Principle for Separating Fluid Mixtures," Ind. Eng. Chem. Fundam., 5(1), 141 (1966).

Wilhelm, R. H., and N. H. Sweed, "Parametric Pumping: Separation of Mixture Toluene and n-Heptane," Science, 159, 522 (1968).

Yang, R. T., Gas Separation by Adsorption Processes, Butterworth, Stoneham, MA (1987)

Zhi, W. X., and P. C. Wankat, "Continuous Mutlicomponent Parametric Pumping," Ind. Eng. Chem. Fund., 22(2), 172 (1983).

Manuscript received Aug. 2, 1994, and revision received Dec. 1, 1994 